\title{
Molecular and structural patterns of bone regeneration in surgically created defects containing bone substitutes
}

Ibrahim Elgali $^{\mathrm{a}, \mathrm{e}}$, Kazuyo Igawa ${ }^{\mathrm{a}, \mathrm{e}}$, Anders Palmquist ${ }^{\mathrm{a}, \mathrm{e}}$, Maria Lennerås ${ }^{\mathrm{a}, \mathrm{e}}$, Wei Xia ${ }^{\mathrm{b}, \mathrm{e}}$, Sungjin Choi ${ }^{\mathrm{c}}$, Ung-il Chung ${ }^{\mathrm{c}, \mathrm{d}}$, Omar Omar ${ }^{\mathrm{a}, \mathrm{e}}$ and Peter Thomsen ${ }^{\mathrm{a}, \mathrm{e}^{*}}$

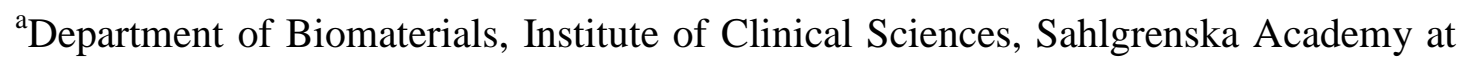
University of Gothenburg, Box 412, SE-405 30 Gothenburg, Sweden

${ }^{\mathrm{b}}$ Applied Materials Science, Department of Engineering Sciences, Uppsala University, Box 534, SE-751 21 Uppsala, Sweden

${ }^{c}$ Division of Clinical Biotechnology, Center for Disease Biology and Integrative Medicine, Graduate School of Medicine, The University of Tokyo, 7-3-1 Hongo, Bunkyo-ku, Tokyo 113-8655, Japan

${ }^{\mathrm{d}}$ Department of Bioengineering, Graduate School of Engineering, The University of Tokyo, 7-3-1 Hongo, Bunkyo-ku, Tokyo 113-8655, Japan

${ }^{\mathrm{e}}$ BIOMATCELL VINN Excellence Center of Biomaterials and Cell Therapy, Box 412, SE-405 30 Gothenburg, Sweden

*Correspondence to: Peter Thomsen; PhD, Professor

Department of Biomaterials, Institute of Clinical Sciences, Sahlgrenska Academy at University of Gothenburg, Box 412, SE-405 30 Gothenburg, Sweden

Landline: + 46 (0) 317862950

Fax: + $46(0) 317862941$

email: peter.thomsen@biomaterials.gu.se

The authors declare no conflict of interest. 


\begin{abstract}
Several biomaterials have been introduced for bone augmentation. However, information is lacking about the mechanisms of bone regeneration and/or integration of these materials in the recipient bone. This study aimed to determine the molecular and structural events in bone defects after augmentation with synthetic tetrapodshaped calcium phosphate (Tetrabone; TetraB) compared with natural deproteinized bovine bone (DBB). Defects were created in the epiphyses of rat femurs and filled with TetraB or DBB or left empty (Sham). After 3, 6, 14 and 28d, samples were harvested for histology, histomorphometry, ultrastructure and gene expression analyses. At 3d, higher expressions of bone formation (ALP and OC) and remodeling (CatK) genes were detected in TetraB compared with DBB and Sham. Downregulation of bone remodeling genes (TRAP and CatK) was detected in DBB as compared to Sham after 14d. Histomorphometry at 6 and 14d demonstrated greater bone contact with the granules in TetraB. At $28 \mathrm{~d}$, a larger bone area per defect was found in TetraB. The present experiments show that a synthetic substitute, consisting of $\alpha$-tricalcium and octacalcium phosphates, induces early osteogenic and osteoclastic activities and promotes bone formation in trabecular bone defects.
\end{abstract}

Keywords: $\alpha$-tricalcium phosphate; octacalcium phosphate, deproteinized bovine bone, gene expression; in vivo; ultrastructure 


\section{Introduction}

Over the last few years, the need for bone augmentation in skeletal surgical operations has increased. The application of bone grafts to promote bone regeneration has become a routine procedure, especially when the endogenous regenerative mechanisms are insufficient to achieve complete healing, e.g. large bone defects. In clinical practice, autografts have been regarded as a golden standard for bone repair and substitution, followed by allografts, where both have great osteoinductive properties. However, problems related to limitations, morbidity, rejection and/or infections are still drawbacks [1, 2]. Many efforts have been made to develop synthetic grafting materials as an alternative option for bone substitution.

Ceramic materials, based on calcium phosphate, are the most developed and clinically used materials, given their relative biocompatibility and similarity to the bone mineral composition. The state-of-the-art material is a natural deproteinized bovine bone (DBB) with good experimental and clinical documentation and follow-up [3-5]. On the other hand, several synthetic forms, showing variable biological behaviors and bone tissue response, have been suggested $[6,7]$. One example of synthetic materials is a recently developed bone substitute consisting of alpha-tricalcium phosphate $(\alpha-$ TCP) and developed as tetrapod-shaped granules (Tetrabone; TetraB) [8]. The granules underwent additional treatment to recrystallize their surfaces into octacalcium phosphate (OCP) and, when implanted in vivo, promoted greater bone formation in comparison to beta-tricalcium phosphate [8]. Whereas data are available on the overall bone formation in conjunction with natural and synthetic substitutes, the molecular mechanisms evoked by these materials, and their relationship to the degree of bone formation, have not been characterized. 
Bone healing and regeneration is a complex sequence of events that involves the modulation of the local microenvironment with cytokines and growth factors, as well as the recruitment and differentiation of different cell populations [9]. Inflammatory cytokines, such as tumor necrosis factor-alpha (TNF- $\alpha$ ) and interleukin-1 beta (IL$1 \beta$ ), are integral molecules during the early phase of bone healing and may positively or negatively affect the regeneration process [10]. Osteogenic differentiation, on the other hand, is determined with specific differentiation molecules, such as alkaline phosphatase (ALP) and osteocalcin (OC). Other important biological molecules are those involved in bone remodeling, such as cathepsin $\mathrm{K}$ (CatK) and tartrate-resistant acid phosphatase (TRAP), which are important for bone maturation. It is hypothesized that, during defect augmentation, these events are influenced by the presence, and the properties, of the substitute, which may in turn affect the overall bone formation and its structural and ultrastructural characteristics. In addition, it is also possible that biological processes, such as bone remodeling, influence the substitute material, e.g. persistence or degradation. Nevertheless, to the authors' knowledge, these hypotheses have not yet been characterized in vivo. The main questions to be addressed are (i) How do the different bone substitute materials influence the temporo-spatial gene expression in cells in defects in comparison to unaugmented defects? (ii) What is the relationship between molecular activities and the amount and structure of the newly formed bone in response to the different substitutes? (iii) Does the expression of genes denoting inflammation, bone formation and bone remodeling correlate and coordinate in specific ways with and without the substitute material? Furthermore, information about osteoclastic activities during bone augmentation may cast some light on the controversy relating to the biodegradation and/or resorbability of natural bovine and synthetic substitutes. 
The general aim of the present study was to compare the differences in biological response between synthetic $\alpha$-tricalcium and octacalcium substitute and the clinical golden standard, deproteinized bovine bone substitute. The following specific aims were formulated: (i) to investigate the spatial and temporal pattern of gene expression regulating inflammation, bone formation and remodeling in surgically created defects with and without augmentation with natural or synthetic bone substitutes and (ii) to relate the molecular patterns associated with the natural and synthetic substitutes and unaugmented defects to the morphology and ultrastructure of the regenerated bone.

\section{Materials and Methods}

\section{Material preparation}

The fabrication process of tetrapod-shaped synthetic bone substitute has been described in a previous study [8]. In short, the binder, a mixture of olefin resin (55\%), wax $(30 \%)$ and plasticizing materials (15\%), was prepared. $\alpha$-TCP powder (Taihei Chemical Industrial Co., Tokyo, Japan; 60\%) and binder (40\%) were mixed for 30 min and the $\alpha$-TCP compound was injected into tetrapod-shaped injection molds with a size of $1 \mathrm{~mm}$ using an injection-molding machine (J35AD; Japan Steel Works, Tokyo, Japan). Molded $\alpha$-TCP compounds were degreased at $500^{\circ} \mathrm{C}$ for $1 \mathrm{~h}$ and calcined at $700^{\circ} \mathrm{C}$ for $1 \mathrm{~h}$. They were soaked in $0.2 \mathrm{M}$ succinic acid for $24 \mathrm{~h}$ to form OCP and rinsed twice with distilled water. They were dried under reduced pressure and sterilized by electron beam irradiation at $25 \mathrm{kGy}$.

The natural substitute was purchased as anorganic deproteinized bovine bone with a particle size of 250-1000 $\mu \mathrm{m}$ (Bio-Oss; Geistlich Pharma, Wolhusen, Switzerland).

\section{Material characterization}


For X-ray diffraction analysis (XRD), TetraB and $\mathrm{DBB}$ were ground into powder and analyzed using a Bruker D8 X-ray diffractometer (Bruker, Karlsruhe, Germany) with a CuKa radiation source at $40 \mathrm{kV}$ and $40 \mathrm{~mA}$, scanning from 3 to $80^{\circ}$.

To evaluate the material shape and surface morphology, scanning electron microscopy (Leo Ultra 55 FEG SEM) was performed operating at $5 \mathrm{kV}$ using a secondary electron detector. Magnifications between 50 and 100,000 times were used to assess the macroscopic geometry of the materials and the micron and submicron surface morphology.

\section{Animal surgery}

The animal experiments were approved by the University of Gothenburg, local ethics committee for laboratory animals (Dnr 301-2009). A total of 84 two-month-old female Sprague-Dawley rats underwent surgery in the present study. All the rats were allowed a one-week acclimatization period and free movement with food and water ad libitum before surgery. At surgery, the animals were anesthetized using an isofluorane (Isoba Vet, Schering-Plough, Uxbridge, UK) gas inhalation with a Univentor 400 anesthesia unit (Univentor, Zejtun, Malta). Anesthesia was maintained by the continuous administration of isofluorane via a mask. The distal aspect of the femur was cleaned with $5 \mathrm{mg} / \mathrm{mL}$ chlorhexidine and $70 \%$ ethanol. Subsequently, the site was shaved and a longitudinal incision was made, followed by skin and periosteal reflection with a blunt instrument. A defect was made in each femoral epiphysis (trabecular bone region) using a trephine with a $2.3 \mathrm{~mm}$ internal diameter and $3 \mathrm{~mm}$ penetration depth under profuse irrigation with $\mathrm{NaCl} 0.9 \%$. Each defect was filled with either tetrapod-shaped bone substitute (TetraB) or deproteinized bovine bone substitute (DBB) or left empty (Sham). Substitute and Sham sites were randomized, ensuring equal distribution and rotation among the animals and between right and left 
locations. The granules from either type were installed one by one in each defect until the defect was completely filled. The subcutaneous layer of the wound was closed with resorbable polyglactin sutures (4-0, Vicryl) and the skin was closed with intracutaneous, resorbable monocryl sutures (4-0). Postoperatively, each rat received an analgesic (Temgesic $0.03 \mathrm{mg} / \mathrm{kg}$, Reckitt \& Coleman, Hull, UK) by subcutaneous injection. The retrieval procedure was performed at 3, 6, 14 and $28 \mathrm{~d}$ for gene expression, histology, histomorphometry and ultrastructural analyses. Animals were sacrificed using an overdose of barbiturate (Mebumal, ACO Läkemedel AB, Solna, Sweden). The skin was reopened and the bone defects filled with the TetraB or DBB or the unfilled Sham ones were identified. Sites intended for gene expression analysis were retrieved using a $2.3 \mathrm{~mm}$ trephine. The samples were immediately preserved in tubes containing RNAlater $(\mathrm{n}=8)$. Sites designated for histology and ultrastructure were harvested en bloc using dental disc. The harvested samples were then immersed in formalin $(n=6)$.

\section{Quantitative polymerase chain reaction}

Total RNA from the trephine-retrieved samples was extracted using an RNeasy® Mini kit (QIAGEN GmbH, Hilden, Germany). Prior to extraction, samples were homogenized in phenol/guanidine-based Qiazol lysis reagent using $5 \mathrm{~mm}$ stainless steel beads (QIAGEN GmbH, Hilden, Germany) and TissueLyser (QIAGEN GmbH, Hilden, Germany). Following the addition of chloroform, the samples were centrifuged at $12,000 \mathrm{~g}$ for $15 \mathrm{~min}$ and the aqueous phase was used for subsequent RNA extraction. DNAse treatment was performed in order to eliminate any contamination by genomic DNA. Reverse transcription was carried out using an iScript cDNA Synthesis Kit (Bio-Rad, Hercules, USA). RNA was normalized to 50 $\mathrm{ng} / \mu \mathrm{l}$ before the reverse transcription. The design of primers for six target genes, 
tumor necrosis factor- $\alpha$ (TNF- $\alpha$ ), interleukin-1 $\beta$ (IL-1 $\beta)$, alkaline phosphatase (ALP), osteocalcin (OC), tartrate resistant acid phosphatase (TRAP) and cathepsin K (CatK), as well as five putative reference genes, was performed using the Primer3 web-based software [11]. The expression profiles of reference genes were evaluated by geNorm [12] and Normfinder [13] software, in order to determine the best reference gene for normalization. Design parameters were adjusted to minimize the formation of artifact products and to be able to use an annealing temperature in the PCR of about $60^{\circ} \mathrm{C}$. Primers were designed to yield short amplicons (preferably shorter than $200 \mathrm{bp)}$ ) and to function well with SYBR Green I fluorescent dye for the detection of the PCR products in real time. Real-time PCR was performed in duplicate using the Mastercycler ep realplex (Eppendorf, Hamburg, Germany) in $10 \mu 1$ reactions. The cycling conditions were $95^{\circ} \mathrm{C}$ for $10 \mathrm{~min}$, followed by 45 cycles of $95^{\circ} \mathrm{C}$ for $20 \mathrm{~s}$, $60^{\circ} \mathrm{C}$ for $20 \mathrm{~s}$ and $72^{\circ} \mathrm{C}$ for $20 \mathrm{~s}$. The fluorescence was read at the end of the $72^{\circ} \mathrm{C}$ step. Melting curves were recorded after the run by a stepwise temperature increase $\left(1^{\circ} \mathrm{C} / 5 \mathrm{~s}\right)$ from 65 to $95^{\circ} \mathrm{C}$. Both geNorm and Normfinder identified the genes hypoxanthine phosphoribosyltransferase 1 (HPRT1) and tyrosine 3monooxygenase/tryptophan 5-monooxygenase activation protein, zeta polypeptide (YWHAZ) as the most stable ones in the present experimental study. Quantities of target genes were normalized using the mean Ct values of HPRT1 and YWHAZ. The normalized relative quantities were calculated using the delta $\mathrm{Ct}$ method and 90\% PCR efficiency $\left(\mathrm{k}^{*} 1.9^{\Delta \mathrm{Ct}}\right)[14]$.

\section{Histology and histomorphometry}

After fixation, the blocks were dehydrated in ascending series of ethanol, followed by embedding in acrylic resin (LR White) (London Resin Company Ltd, Berkshire, UK). The embedded specimens were cut along the long axis of the defect using a diamond 
saw. Subsequently, one half of each specimen was used to produce ground sections prepared by sawing and grinding (Exakt Apparatebau GmbH \& Co, Norderstedt, Germany). The sections were made with a thickness of $10-20 \mu \mathrm{m}$ and stained with $1 \%$ toluidine blue. The sections were then coded and evaluated blindly under a light microscope (Nikon Eclipse E600) for histological and histomorphometric analyses. The qualitative histological evaluation was made in an optical microscope (Nikon Eclipse E600; Nikon Ltd., Tokyo, Japan), using image software (ACT-1; Nikon Ltd., Tokyo, Japan). A quantitative histomorphometry analysis was performed in the same microscope using analytical software (Easy Image Measurement 2000; Technooptik AB, Huddinge, Sweden).

The histomorphometric analysis was performed using 10x objective. In each defect, the area percentages of granules (TetraB or DBB), as well as the newly formed bone (TetraB, DBB or Sham), were calculated with respect to the total defect area. Furthermore, the percentage of bone-granule contact (TetraB or DBB) was calculated with respect to the total circumference of the individual granules. This was achieved by performing the measurement using a software grid consisting of nine zones, which covered the total area of defect. The area of the newly formed bone, the area of granules or the bone-granule contact were separately traced in each zone and percentages of the areas (bone or granules) and the contacts were then calculated from the sum of the zones. The discrimination of the newly formed bone from the natural bovine bone substitute was judged by the absence of osteons/Haversian system, the relatively darker staining and the presence of relatively round and viable osteocytes.

\section{Ultrastructural analysis}


The other halves of the specimens were prepared for electron microscopy. The cut side was polished and the specimen was glued to a regular SEM stub and subsequently coated with a $10 \mathrm{~nm}$ thick conductive coating (AuPd). Back-scatter SEM (BS-SEM) was performed in a Leo Ultra 55 FEG SEM (blab la) operating at 20 $\mathrm{kV}$. A quadrapole detector using Compo mode was used to obtain optimal Z-contrast. Magnifications between 50 and 50,000 were used to evaluate the overview bone response and resolve the interfacial reactions over time. For the $28 \mathrm{~d}$ time point, transmission electron microscopy (TEM) samples across the material-bone interface were prepared using focused ion beam (FIB, FEI Strata DB235) in-situ lift out [15]. The samples were thinned to a thickness of approximately $100 \mathrm{~nm}$ using a decreasing ion beam current. TEM analyses were performed in a FEI Tecnai TEM/STEM, using both bright-field TEM and high-angle annular dark-field STEM (HAADF-STEM).

\section{Statistics}

For histomorphometry and gene expression analyses, statistical comparisons were made between the three groups (Sham, DBB and TetraB) and between the time periods for each group. A non-parametric Kruskal-Wallis test was used to identify statistical differences between the groups or time periods. Whenever a statistical difference was found, the Mann-Whitney test was used to determine the statistical difference between two groups. Spearman's correlation analysis was performed between the expression levels of the analyzed genes in each defect type and at the 6 , 14 and 28d time points. Analyses were made using SPSS Version 10 software (SPSS, Inc., Chicago, USA) and the significance was set at $\mathrm{p}<0.05$. The data are presented as the mean \pm standard error of the mean.

\section{Results}




\section{Material characterization}

The SEM evaluation of the materials showed that the DBB had an irregular shape, with dimensions varying between a few hundred $\mu \mathrm{m}$ to one mm (Fig. 1A), while the TetraB showed a highly ordered four-legged shape, with dimensions of the struts of a couple of hundred $\mu \mathrm{m}$ in diameter and a length of $600 \mu \mathrm{m}$, creating a global size of one $\mathrm{mm}$ (Fig. 1B). At higher resolution, smaller granules, 5-20 $\mu \mathrm{m}$ in size, were present on the DBB surface (Fig. 1C), which was otherwise characterized as a solid material on this length scale. At similar magnification, TetraB also showed a solid material with a surface composed of flake-like structures ranging in size from 5-10 $\mu \mathrm{m}$ (Fig. 1D). At high resolution (Fig. 1E, F), a rougher surface was observed for both materials; the DBB was composed of aggregates of particles ranging in size from 400$500 \mathrm{~nm}$, with individual particle sizes of less than $100 \mathrm{~nm}$ in the smallest dimension. The TetraB showed a porous surface between the unordered flake structure. The dimensions of the flakes showed a smallest dimension of approximately $100 \mathrm{~nm}$, with fairly sharp edges.

The XRD analysis (Fig. 2) revealed that the prominent phase of DBB was mainly hydroxyapatite, whereas TetraB revealed several $\alpha$-TCP peaks and, to a lesser extent, peaks that correspond to OCP and hydroxyapatite.

\section{Histology and histomorphometry}

At 6d, both $\mathrm{DBB}$ and TetraB granules appeared well contained within the created defect boundaries (Fig. 3B, C). At this time point, there was no major evident bone formation in the central region of any of the defect groups (Fig. 3A, B, C). The central regions were mainly occupied by soft tissue in the Sham defects (Fig. 3A), whereas the augmented defects were mainly occupied by the DBB (Fig. 3B) or TetraB (Fig. 3C) granules. Peripherally, newly formed osteoid was observed in all defect types. No 
major inflammatory infiltrates were detected in any of the defects. Evaluation at higher magnification (Fig. 4) showed that osteoid was formed in association with cells in the intergranular space. Two major observations distinguished the DBB and TetraB defects from one another. Firstly, the osteoid in the TetraB group (Fig. 4D, E, F) revealed greater proximity and contact with the granule surfaces than that formed in the DBB group (Fig. 4A, B, C). Secondly, areas of partially mineralized bone, indicated by slightly lighter staining than the osteoid, were mainly detected in the TetraB-treated defects (Fig. 4D). Osteoblast seams were commonly observed adjacent to the osteoid areas in all defect groups. Histomorphometry at $6 \mathrm{~d}$ revealed no major difference in the area percentage of the newly formed bone between the three groups, which ranged between $9-11 \%$ of the total defect area (Fig. 7). On the other hand, the analysis revealed a higher percentage of bone-granule contact in the TetraB $(37.4 \pm$ 10.7) compared with the DBB $(5 \pm 1.8 ; \mathrm{p}<0.05)$ (Fig. 7). Measurements of the area percentages occupied by the granules of each material revealed slightly higher values in the TetraB group $(35.4 \% \pm 1.3)$ compared with the equivalent for DBB $(28 \pm 4.5)$, but the difference was not statistically significant.

At 14d, a substantial amount of bone had formed, extending from the periphery of the defect and filling the central region in all groups (Fig. 3D, E, F). In the augmented sites (Fig. 3E, F), the newly formed bone displayed a trabecular pattern and appeared to fill the intergranular spaces. In comparison with 6d, a higher degree of mineralization of the newly formed bone was observed for all groups, as judged by the decreased intensity of the toluidine blue staining and the presence of osteocytes (Fig. 5). Moreover, as judged by the staining, the newly formed bone in the TetraB group appeared more mineralized than that observed in the DBB group (Fig. 5A, E). In the TetraB group, the newly formed bone showed a greater degree of contact with 
the granule surface than that in the DBB (Fig. 5 B, F). Furthermore, the interface between the newly formed bone and the TetraB granules frequently revealed a bladelike appearance (Fig. $5 \mathrm{H}$ ). Osteoblast seams and osteoclast-like cells were frequently detected, in many cases in connection with the granule surfaces of either material (Fig. 5 C, D, G, I). In addition, surface depressions and irregularities, indicating resorption areas, were frequently detected in association with the osteoclast-like cells. These resorption areas were observed on the surfaces of both bone and granules in the TetraB defects (Fig. $5 \mathrm{G}$ ), whereas a similar finding was only made for bone surfaces in the DBB defects (Fig. 5 C). In comparison with 6d, histomorphometry revealed a significant increase in the proportion of newly formed bone for all defect types (Fig. 7). In this period, albeit not statistically significant, Sham sites revealed a slightly higher bone area percentage $(31.8 \% \pm 2.2)$ compared with DBB $(24.6 \pm 1)$ and TetraB $(26.6 \pm 3.4)$ (Fig. 7). After $14 d$, the bone contact with the TetraB granules $(90 \%)$ was significantly higher than that with DBB (60\%) (Fig. 7). For both defect types, the percentage of bone-granule contact revealed a significant increase from that observed after $6 \mathrm{~d}$. No major difference in the granule area percentages was observed between the TetraB and DBB (31.3 \pm 2 and $26 \pm 1.5$ respectively). No significant differences in granule area percentage between $6 \mathrm{~d}$ and $14 \mathrm{~d}$ were detected.

At 28d, the bone formed in the Sham site showed considerable resorption, leaving a few trabeculae of mature bone, bordered endosteally by the bone marrow and periosteally by the overlying soft tissue (Fig. 3G). In the Sham defects, the original bone contour was not achieved. The soft tissue on the periosteal side of the Sham defects displayed downgrowth into the defect area (Fig. 3G). In contrast, the augmented sites appeared to maintain a high degree of mature bone after $28 \mathrm{~d}$. In both the DBB- and TetraB-augmented defects, the mature bone extended to the top of the 
defects, regaining the original level of the bone at these sites (Fig. 3H, I). A larger amount of mature bone appeared to fill the intergranular spaces in the TetraB group compared with the DBB (Fig. 3H, I, Fig. 6A, D). Active remodeling sites were encountered, characterized by newly formed osteoid, osteoblast seams and osteoclastlike cells. This observation was more commonly made in the TetraB group (Fig. 6E, F). Histomorphometric analysis at $28 \mathrm{~d}$ revealed a significantly higher bone area percentage in TetraB defects $(37.5 \pm 3.9)$ compared with the Sham $(21.2 \pm 4)$ and the DBB $(24 \pm 2.2)$ (Fig. 7). On the other hand, the bone-granule contact was significantly decreased in the TetraB sites and unchanged in the DBB sites, resulting in a $76.7 \pm 4.5$ and $67 \pm 1.5$ contact percentage, respectively. In this period, there was no significant difference in the percentage of bone-granule contact between the TetraB and DBB. Like the previous time periods, no major changes in the percentages of the granule areas were detected, in either TetraB $(29.3 \pm 4)$ or DBB $(27.9 \pm 4.6)$ defects.

\section{Ultrastructure}

At the early time points, 3 and 6d, limited mineralized tissue had formed within the defects in the Sham (data not shown) and DBB (Fig. 8A, C) groups. For TetraB, some mineralization had occurred in association with the surface (Fig. 8B, D). After 14d, bone formation around and in direct contact with both materials was evident (Fig. 9). Less mature tissue was observed towards the center of the defect, as judged by less organization and less contrast. Osteocyte lacunae were frequently seen close to both materials. At higher resolution, bone had formed around the flakes on the TetraB surface, but, further into the surface coating, low contrast was observed, indicating a porous zone (Fig 9D). At 28d (Fig. 10), the bone tissue had matured around both materials as compared to the $14 \mathrm{~d}$ time point. Osteocytes were present close to the 
materials, at a distance of $2-5 \mu \mathrm{m}$ from the surface. The bone contoured the surface irregularities and topography, while a porous zone of a couple of $\mu \mathrm{m}$ was still present in the subsurface of the TetraB samples. This was further confirmed by TEM, where a hollow zone around the flakes was present between the bone tissue and the bulk grains of the material (Fig. 10F). The DBB showed intimate contact with bone at all the different resolutions within the study. The collagen fibers ran parallel to the surfaces, indicated by the collagen banding (TetraB; Fig. 10F) or the transversely cut collagen fibers (DBB; Fig. 10E).

\section{Gene expression}

Comparisons were made of gene expression in bone samples harvested from the defects after different healing periods. In this way, a selection of targeted genes involved in inflammation, bone formation and bone remodeling was analyzed at 3, 6, 14 and $28 \mathrm{~d}$ after the defect creation with and without augmentation. The results of these gene expression analyses are summarized in Fig. 11. In addition, the relationships between the expressions of the targeted gens in Sham, DBB or TetraB defects, in the different time periods, were evaluated and the data are presented in Table I.

\section{Gene expression of proinflammatory cytokines}

At $3 \mathrm{~d}$, the expression levels of TNF- $\alpha$ and IL-1 $\beta$ were downregulated about two-fold in the TetraB sites compared with the Sham, although the differences were not statistically significant. Furthermore, no major difference between DBB and Sham was detected at this time. At $6 \mathrm{~d}$, all the defects revealed comparable levels of these proinflammatory cytokines. Later, at $14 \mathrm{~d}$, a two-fold lower expression of IL-1 $\beta$ was demonstrated in the DBB in comparison to the Sham $(\mathrm{p}<0.05)$ and to the TetraB $(\mathrm{p}>$ 
0.05). Similar to $6 \mathrm{~d}$, no major differences between the three groups were detected after $28 \mathrm{~d}$ with respect to the expression of the proinflammatory cytokines.

The temporal expression pattern of both cytokines revealed high peaks for all groups at $3 \mathrm{~d}$. Thereafter, there were general downregulations in all groups from $3 \mathrm{~d}$ to $6 \mathrm{~d}$. The downregulations were statistically significant in all groups with respect to TNF- $\alpha$, whereas they were only significant in the DBB defects with respect to IL-1 $\beta$. From $6 \mathrm{~d}$ to $14 \mathrm{~d}$, there was a slight increase in the expression of TNF- $\alpha$, mainly in the Sham defects. On the other hand, IL-1 $\beta$ revealed temporal increases from $6 \mathrm{~d}$ to $14 \mathrm{~d}$ in the Sham $(\mathrm{p}<0.05)$ and the TetraB $(\mathrm{p}>0.05)$ but not in the DBB. No major temporal changes were observed in the expression of both cytokines from $14 \mathrm{~d}$ to $28 \mathrm{~d}$, in all the groups.

\section{Gene expression of bone formation markers}

Gene expression analysis at $3 \mathrm{~d}$ revealed significantly higher levels of ALP and OC in the TetraB group. In this early period, TetraB revealed about a 2-fold higher expression of ALP in comparison to Sham $(\mathrm{p}<0.05)$ and to DBB $(\mathrm{p}>0.05)$. At the same time, the expression of OC was 3- and 13-fold significantly higher in TetraB compared with Sham and DBB, respectively. Apart from the slightly lower expression of OC in the DBB group at $14 \mathrm{~d}$, there were no major differences between the groups at the remaining time points with respect to the osteogenic gene expression.

The temporal expression pattern showed the upregulation of both ALP and OC from $3 \mathrm{~d}$ to $6 \mathrm{~d}$ in the Sham and the DBB groups, whereas no significant change was found for TetraB. From $6 \mathrm{~d}$ to $14 \mathrm{~d}$, there was a general upregulation in the expression of both ALP and OC in all the groups and these upregulations were statistically significant in TetraB (for ALP expression) and in Sham and TetraB (for OC expression). 
Thereafter, from $14 \mathrm{~d}$ to $28 \mathrm{~d}$, there was a significant downregulation in the expression

\section{Gene expression of bone resorption markers}

In general, a similar expression profile was observed for TRAP and CatK. At 3d, both osteoclastic genes were expressed at higher levels (1.5-2.5-fold) in TetraB defects compared with Sham and DBB defects. However, these higher levels only attained statistical significance with respect to CatK. Both genes were expressed at similar levels among the three defect types at the $6 \mathrm{~d}$ time point. At $14 \mathrm{~d}$, the expression of CatK and TRAP showed similar levels for Sham and TetraB defects. At this time point, both genes revealed about a 1.5 -fold lower expression in DBB defects compared with Sham and TetraB, but the difference was only statistically significant for TRAP expression when compared with Sham. No major differences between the groups were detected at $28 \mathrm{~d}$.

The temporal expression of CatK and TRAP revealed a general upregulation from $3 \mathrm{~d}$ to $6 \mathrm{~d}$ in all the groups. From $6 \mathrm{~d}$ to $14 \mathrm{~d}$, the temporal expression of both genes was further increased in Sham and TetraB, whereas it was maintained at similar levels in DBB. Thereafter, from $14 \mathrm{~d}$ to $28 \mathrm{~d}$, both genes demonstrated a downregulation in all defect types.

\section{Correlation analysis of gene expression}

The results from the correlation analysis of the different genes are presented in Table I.

At $3 \mathrm{~d}$, the Sham defects showed a significantly negative $(r=-0.94 ; \mathrm{p}=0.004)$ and positive $(r=0.83 ; \mathrm{p}=0.04)$ correlation for TNF- $\alpha$ with OC and TRAP, respectively. On the other hand, in the DBB and TetraB defects, a significant association was only 
registered as a positive correlation between the osteoclastic/remodeling genes, TRAP and CatK, $(r=0.83 ; p=0.04)$ and $(r=0.96 ; 0.0004)$, respectively.

At 6d, a significantly positive correlation between osteoclastic/remodeling genes (TRAP and CatK) was detected in the three defects (Sham, DBB and TetraB). At this time point, TetraB defects revealed significantly positive relationships between the bone formation gene (OC) and the remodeling genes, TRAP and CatK, with correlation coefficients of $\mathrm{r}=0.89(\mathrm{p}=0.006)$ and $\mathrm{r}=0.79(\mathrm{p}=0.04)$, respectively. Furthermore, the bone formation gene (ALP) in the TetraB defects demonstrated a positive correlation with the inflammatory cytokine $(\mathrm{TNF}-\alpha)$ at a level of $r=0.79(p=$ $0.04)$.

At $14 \mathrm{~d}$, a positive correlation between inflammatory cytokines (TNF- $\alpha$ and IL-1 $\beta$ ) was detected in all defect types. At this time, whereas this was the only correlation detected in the Sham defects, there were several correlations between the different genes in the DBB and TetraB defects. In both defects, there was a positive correlation between the osteoclastic/remodeling genes (TRAP and CatK). Moreover, positive correlations were demonstrated for both osteoclastic/remodeling genes (TRAP and CatK) with the inflammatory cytokines (TNF- $\alpha$ and IL-1 $\beta$ ), in both defects. The highest level of significance was registered between IL-1 $\beta$ and CatK, with $r=1$ and $r$ $=0.96(\mathrm{p}<0.0005)$ in the DBB and TetraB, respectively.

At 28d, significantly positive correlations were found between the proinflammatory cytokines (TNF- $\alpha$ and IL-1 $\beta$ ) and between the osteoclastic/remodeling genes (TRAP and CatK) in all defects. At this time, the Sham defects showed two types of significant correlation. The first was a positive correlation for the bone formation gene (ALP) with the inflammatory cytokine (TNF- $\alpha, r=0.89 ; \mathrm{p}=0.02)$ and the second was the positive correlation for the other bone formation gene (OC) with both 
osteoclastic/remodeling genes (TRAP and CatK, $r=0.89 ; p=0.02$ ). In the DBB and TetraB defects, significantly positive correlations were registered between the bone formation genes (ALP and OC) and for the bone formation gene (ALP) with the osteoclastic gene (CatK). In addition, the TetraB defects demonstrated significantly positive correlations for the inflammatory cytokine (TNF- $\alpha$ ), with the bone formation gene (ALP, $r=0.93 ; p=0.002)$ and the osteoclastic/remodeling gene $(\mathrm{CatK}, \mathrm{r}=0.79$; $\mathrm{p}=0.04)$ and for the other inflammatory cytokine (IL-1 $)$ with the osteoclastic remodeling gene (TRAP, $r=0.82 ; p=0.02)$ and $($ CatK, $r=0.79 ; p=0.04)$.

\section{Discussion}

The present study describes the relationship between the molecular determinants of bone regeneration and the kinetic changes in the amount and structure of the regenerated bone during defect augmentation with apatite-based bone substitutes. The main findings were that the presence of the bone substitute, irrespective of type, significantly modulates the level and the coordination of the molecular activities, resulting in the different level and distribution of bone from that seen in unaugmented defects. Furthermore, the molecular and structural events of bone regeneration are substantially affected by the differences in the crystallinity, structure and/or surface morphology of the two substitute materials.

During the early phase of bone defect healing, the trigger of osteogenic differentiation and bone formation corresponds to the diminution of the acute inflammation induced by the surgical trauma. The present findings relating to the early global downregulation of proinflammatory gene expression, in the presence or absence of the substitute materials, indicate that both natural and synthetic substitutes do not disrupt the biological switch from inflammation to bone regeneration or, at least, do not prolong the inflammatory phase after implantation. 
One major observation in the present study was the early, increased level of bone contact with the surface of the synthetic substitute in parallel with the higher expression of bone formation and remodeling genes and the downregulation of proinflammatory genes. This relationship has been previously reported in similar experimental designs, but during titanium implant osseointegration [16, 17]. Oxidized implants associated with higher bone formation and remodeling and lower proinflammatory gene expressions induced a higher level of bone contact than equivalent implants with machined surfaces [17]. Apart from the differences in the design and the bulk materials, both findings illustrate a rapid, intense and confined pro-osteogenic microenvironment established between the material surface and the adherent cells. In contrast to osseointegration, the consequence of increased bonematerial contact during augmentation with the particulate materials remains to be determined.

Another interesting finding was the relationship between the early gene expression and the ultrastructure of the bone interface and the synthetic granules. The early, coupled upregulation and the strong correlation of bone formation and bone remodeling gene expression were associated with the early, ultrastructural detection of, at least partially, mineralized bone in response to the synthetic substitute. Remodeling is an essential process for the maturation of the bone that forms early during healing; however, this process has traditionally been regarded as a late maturation stage, which takes place over several weeks. The present results suggest that an accelerated remodeling phase takes place in conjunction with early bone formation in response to specific properties of the synthetic substitute.

The impact of proinflammatory cytokines during bone regeneration in vivo is highly controversial. For instance, there are many indications that TNF- $\alpha$ plays different 
roles during different periods of bone healing and regeneration [18-20]. They include effects on mesenchymal stem cell migration and osteogenic and osteoclastic cell differentiation [21-23]. During the $3 \mathrm{~d}$ period, even though there were no significant differences in the expression of TNF- $\alpha$ in the three defects, the higher trend in the expression of TNF- $\alpha$ in the unaugmented Sham defects was in parallel with a negative correlation with the expression of osteogenic genes. This observation suggests a negative role for TNF- $\alpha$ at the high level of expression detected in the unaugmented defect at the $3 \mathrm{~d}$ period. Interestingly, at the late time period ( $14 \mathrm{~d}$ and $28 \mathrm{~d})$, the lower level of expression of TNF- $\alpha$ was positively related to the osteogenic genes. Although no direct conclusions can be derived from these relationships, possible explanations can still be suggested. Firstly, TNF- $\alpha$ at a high level of expression may negatively affect the osteogenic activity and bone formation in vivo. This assumption is supported by previous in vitro data, showing that TNF- $\alpha$ inhibits mineralization and osteogenic gene expression $[24,25]$. Secondly, TNF- $\alpha$ at a low level of expression may positively influence the osteogenic gene expression during the late period of bone regeneration in vivo. This latter assumption is supported by previous in vitro studies showing that TNF- $\alpha$ increases the matrix mineralization and osteogenic gene expression [23]. Whether the level of expression or the healing time period or both are the most important factor when it comes to determining the osteogenic impact of TNF- $\alpha$ would require further investigation.

Histologically, the bone response to deproteinized bovine bone has been previously investigated [26, 27]. The material has been considered to be osteoconductive [28]. However, there is limited information about the plausible mechanism of bone regeneration in association with $\mathrm{DBB}$. One major debate relates to the resorbability of the natural material when introduced into the biological environment of bone defects. 
Whereas several studies described the presence of osteoclasts and osteoclast-like cells on the surface of the DBB $[29,30]$, other studies revealed that DBB is persistent and undergoes minimum resorption [5, 31, 32]. Clinically retrieved samples showed the presence of DBB granules after 11 years in a volume comparable to that when it was implanted [33]. One main finding in the present study was the downregulation of osteoclastic genes, which may provide a plausible explanation for the low resorptive capacity of the DBB. The histological findings in the present study are in line with this assumption. At the $14 \mathrm{~d}$ period, osteoclast-like cells were detected on the surfaces of both materials, but, after $28 \mathrm{~d}$, the appearance of these cells was more evident in association with the surface of the synthetic substitute. Furthermore, at the $14 \mathrm{~d}$ period, a sign of resorption was mainly observed on the bone side facing the osteoclast-like cells associated with DBB, whereas the cells at TetraB were associated with resorption areas, on surfaces of both bone and granules. Moreover, at 28d, the osteoclast-like cells in TetraB-augmented defects were commonly accompanied by osteoblast seams, suggesting active remodeling sites at the immediate contact with the synthetic substitute.

The XRD data showed that the main crystallinity phase of DBB is hydroxyapatite, which is generally assumed to be the chemically most stable phase of calcium phosphates. Furthermore, the process of deproteinization of bovine bone, including the heat and chemical treatment, may result in increased HA crystallinity, which may make it further inclined towards lower solubility. On the other hand, the surface of the TetraB was chemically treated to produce octacalcium phosphate (OCP) crystallinity, as indicated by the XRD and the ultrastructural surface analyses. The present findings that the osteoclast-like cells produced signs of resorption on both the bone and the material sides, in conjunction with the OCP-containing substitute, and only on the 
bone side in the HA-containing DBB substitute are in accordance with previous findings reported by Kamakura and co-authors [34]. In the latter ultrastructural study in vivo, multinucleated cells associated with OCP were able to form a clear zone and ruffled border in the interface between bone and the granule surface, whereas only a clear zone developed in association with HA. These findings were associated with the frayed appearance of both bone and granule sides in the OCP group and only the bone side in the HA. The present study indicates that the resorptive activity of the osteoclast is down-tuned at the molecular level, where the expressions of major osteoclastic enzymes are significantly downregulated in response to the HAdominated DBB.

Using histology, the appearance of cells with an osteoclastic phenotype in association with OCP granules has been previously reported $[35,36]$. The present morphological and molecular findings show the upregulation of osteoclastic activity at 3 and $14 \mathrm{~d}$ and the frequent appearance of osteoclast-like cells in association with the OCPcontaining substitute at 14 and $28 \mathrm{~d}$. Taken together, these observations suggest major interactions between the osteoclasts and the surface of the OCP granules, leading to the prompt mineralization of the early formed bone in direct contact with the granules and ultimately to an increased amount of mature bone forming in the defects. The proosteogenic potential of osteoclasts may involve different mechanisms: the delivery or activation of latent growth factors released from bone sites during the resorption process [37-39]; the provision of pro-osteogenic signals via cell-to-cell contact [40] or via the release of active pro-osteogenic factors [41-43]. Furthermore, additional mechanisms can be considered based on the present in vivo findings of increased osteoclastic activity at the surface of the synthetic substitute and on previous in vitro findings, showing the ability of osteoclastic cells to resorb different calcium 
phosphate substrates [44-47]. Mechanisms of this kind imply that it is possible that osteoclasts are able to condition the surface of granules or expose the surface features that have been biologically coated after implantation in the recipient sites. This surface conditioning and/or exposure may influence the osteoprogenitors and osteoblasts, upregulating osteogenic gene expression and increasing the level of bone formation and degree of mineralization.

The exact mechanism by which DBB downregulates osteoclastic activity is unclear. The main downregulation of osteoclastic gene expression in the DBB appeared to take place during the $14 \mathrm{~d}$ period. This period in the present animal model corresponds to the phase of active bone remodeling where the early-formed woven bone is remodeled into mature bone. Proinflammatory cytokines, such as TNF- $\alpha$ and IL-1 $\beta$, are considered important for this phase of bone healing. Studies of a similar animal model showed that, after the downregulation of the initial proinflammatory phase, the expressions of proinflammatory cytokines increase again during the remodeling phase about two weeks after injury $[10,48]$. This was confirmed in the unaugmented Sham defects, where the levels of both cytokines showed a temporal increase after 14d. Whereas this temporal upregulation was maintained in the TetraB-augmented defects, there was a major downregulation, especially for IL-1 $\beta$, in the DBB-augmented defects. TNF- $\alpha$ and IL-1 $\beta$ bind to respective receptors on osteoclasts and augment their differentiation and resorptive potential $[21,49,50]$. Possible positive roles for these cytokines in osteoclastic activity are also indicated by the strongly positive correlations between the gene expression of these cytokines and the expression of osteoclastic genes at $3 \mathrm{~d}$, in the unaugmented Sham defects, and at $14 \mathrm{~d}$, both in the unaugmented Sham and in the augmented defects. 
The bone that formed after $28 \mathrm{~d}$ appeared as highly mineralized mature bone, indicated by the high contrast at ultrastructural level. The maturation of bone was not limited to any specific substitute type, as the ultrastructural analysis showed well-organized bone, with collagen fibers and numerous osteocytes, in direct connection with the surfaces of either type of the substitutes. On the other hand, the TetraB-augmented defects displayed a larger amount of newly formed bone than the DBB-augmented defects. In fact, it was the unaugmented sham defects that underwent a massive reduction in bone area percentage, underscoring the beneficial effect of the substitute materials.

In the $28 \mathrm{~d}$ time period, no major differences were observed between the defects with respect to the magnitude of gene expression for any of the biological processes. In contrast to the Sham and DBB defects, which showed positive correlations between genes involved in bone formation and remodeling, the TetraB-treated defects exhibited multiple, strong correlations between the genes denoting the different processes (inflammation, bone formation and remodeling). The increased correlations between the different genes may reflect a state of steadiness and coordination between different cells at the site of bone regeneration.

\section{Conclusion}

Under experimental conditions, in contrast to empty defects, both natural and synthetic bone substitute materials promote bone regeneration and the restitution of the surgically created defect. The present gene expression, morphological and ultrastructural data demonstrate that the bovine substitute (DBB) is osteoconductive and elicits low resorption activity. Further, the synthetic substitute (TetraB) induces early osteogenic and osteoclastic activity, resulting in greater bone formation in comparison to the natural substitute (DBB). 


\section{Acknowledgments}

The authors are indebted to Lena Emanuelsson and Birgitta Norlindh for excellent technical assistance during surgery and histological preparation. This study was supported by the Swedish Research Council (K2012-52X-09495-25-3), the BIOMATCELL VINN Excellence Center of Biomaterials and Cell Therapy, the IngaBritt and Arne Lundberg Foundation, ALF/LUA research grant, the Stiftelsen Handlanden Hjalmar Svensson, the Japan Society for the Promotion of Science (JSPS) through the Grants-in-Aid for Scientific Research, the International Core Research Center for Nanobio, Core-to-Core Program and the Japan Science and Technology Agency (JST) through the S-innovation program. IE is the recipient of an international scholarship for $\mathrm{PhD}$ studies provided by the Ministry of Higher Education, Libya. KI is the recipient of an international postdoc grant provided by BIOMATCELL and BIOSUM National Graduate School in Biomaterials and Cell Therapy. The grant providers were not involved in the study design, data acquisition, interpretation, writing and submission of the article. 


\section{References}

[1] Brydone AS, Meek D, Maclaine S. Bone grafting, orthopaedic biomaterials, and the clinical need for bone engineering. Proc Inst Mech Eng H. 2010;224:1329-43.

[2] Eppley BL, Pietrzak WS, Blanton MW. Allograft and alloplastic bone substitutes: a review of science and technology for the craniomaxillofacial surgeon. J Craniofac Surg. 2005;16:981-9.

[3] Lee DZ, Chen ST, Darby IB. Maxillary sinus floor elevation and grafting with deproteinized bovine bone mineral: a clinical and histomorphometric study. Clin Oral Implants Res. 2012;23:918-24.

[4] Schlegel AK, Donath K. BIO-OSS--a resorbable bone substitute? J Long Term Eff Med Implants. 1998;8:201-9.

[5] Schlegel AK, Donath K, Weida S. Histological findings in guided bone regeneration (GBR) around titanium dental implants with autogenous bone chips using a new resorbable membrane. J Long Term Eff Med Implants. 1998;8:211-24.

[6] Hwang JW, Park JS, Lee JS, Jung UW, Kim CS, Cho KS, et al. Comparative evaluation of three calcium phosphate synthetic block bone graft materials for bone regeneration in rabbit calvaria. $J$ Biomed Mater Res B Appl Biomater. 2012;100:2044-52.

[7] LeGeros RZ. Calcium phosphate-based osteoinductive materials. Chem Rev. 2008;108:4742-53.

[8] Choi S, Liu IL, Yamamoto K, Igawa K, Mochizuki M, Sakai T, et al. Development and evaluation of tetrapod-shaped granular artificial bones. Acta Biomater. 2012;8:2340-7.

[9] Bab I, Sela J. Cellular and Molecular Aspects of Bone Repair. In: Sela J, Bab I, editors. Principles of Bone Regeneration. New York: Springer US; 2012. p. 11-41.

[10] Ai-Aql ZS, Alagl AS, Graves DT, Gerstenfeld LC, Einhorn TA. Molecular mechanisms controlling bone formation during fracture healing and distraction osteogenesis. J Dent Res. 2008;87:107-18.

[11] Rozen S, Skaletsky H. Primer3 on the WWW for general users and for biologist programmers. Methods Mol Biol. 2000;132:365-86.

[12] Vandesompele J, De Preter K, Pattyn F, Poppe B, Van Roy N, De Paepe A, et al. Accurate normalization of real-time quantitative RT-PCR data by geometric averaging of multiple internal control genes. Genome Biol. 2002;3:RESEARCH0034. [13] Andersen CL, Jensen JL, Orntoft TF. Normalization of real-time quantitative reverse transcription-PCR data: a model-based variance estimation approach to identify genes suited for normalization, applied to bladder and colon cancer data sets. Cancer Res. 2004;64:5245-50.

[14] Pfaffl MW. A new mathematical model for relative quantification in real-time RT-PCR. Nucleic Acids Res. 2001;29:e45.

[15] Jarmar T, Palmquist A, Branemark R, Hermansson L, Engqvist H, Thomsen P. Technique for preparation and characterization in cross-section of oral titanium implant surfaces using focused ion beam and transmission electron microscopy. $\mathbf{J}$ Biomed Mater Res A. 2008;87:1003-9.

[16] Omar O, Svensson S, Zoric N, Lenneras M, Suska F, Wigren S, et al. In vivo gene expression in response to anodically oxidized versus machined titanium implants. J Biomed Mater Res A. 2010;92:1552-66.

[17] Omar OM, Lenneras ME, Suska F, Emanuelsson L, Hall JM, Palmquist A, et al. The correlation between gene expression of proinflammatory markers and bone 
formation during osseointegration with titanium implants. Biomaterials. 2011;32:37486.

[18] Gerstenfeld LC, Cho TJ, Kon T, Aizawa T, Cruceta J, Graves BD, et al. Impaired intramembranous bone formation during bone repair in the absence of tumor necrosis factor-alpha signaling. Cells Tissues Organs. 2001;169:285-94.

[19] Kon T, Cho TJ, Aizawa T, Yamazaki M, Nooh N, Graves D, et al. Expression of osteoprotegerin, receptor activator of NF-kappaB ligand (osteoprotegerin ligand) and related proinflammatory cytokines during fracture healing. J Bone Miner Res. 2001;16:1004-14.

[20] Mountziaris PM, Mikos AG. Modulation of the inflammatory response for enhanced bone tissue regeneration. Tissue Eng Part B Rev. 2008;14:179-86.

[21] Azuma Y, Kaji K, Katogi R, Takeshita S, Kudo A. Tumor necrosis factor-alpha induces differentiation of and bone resorption by osteoclasts. J Biol Chem. 2000;275:4858-64.

[22] Fu X, Han B, Cai S, Lei Y, Sun T, Sheng Z. Migration of bone marrow-derived mesenchymal stem cells induced by tumor necrosis factor-alpha and its possible role in wound healing. Wound Repair Regen. 2009;17:185-91.

[23] Hess K, Ushmorov A, Fiedler J, Brenner RE, Wirth T. TNFalpha promotes osteogenic differentiation of human mesenchymal stem cells by triggering the NFkappaB signaling pathway. Bone. 2009;45:367-76.

[24] Gilbert L, He X, Farmer P, Boden S, Kozlowski M, Rubin J, et al. Inhibition of osteoblast differentiation by tumor necrosis factor-alpha. Endocrinology. 2000;141:3956-64.

[25] Gilbert L, He X, Farmer P, Rubin J, Drissi H, van Wijnen AJ, et al. Expression of the osteoblast differentiation factor RUNX2 (Cbfa1/AML3/Pebp2alpha A) is inhibited by tumor necrosis factor-alpha. J Biol Chem. 2002;277:2695-701.

[26] Klinge B, Alberius P, Isaksson S, Jonsson J. Osseous response to implanted natural bone mineral and synthetic hydroxylapatite ceramic in the repair of experimental skull bone defects. J Oral Maxillofac Surg. 1992;50:241-9.

[27] Tapety FI, Amizuka N, Uoshima K, Nomura S, Maeda T. A histological evaluation of the involvement of Bio-Oss in osteoblastic differentiation and matrix synthesis. Clin Oral Implants Res. 2004;15:315-24.

[28] Traini T, Valentini P, Iezzi G, Piattelli A. A histologic and histomorphometric evaluation of anorganic bovine bone retrieved 9 years after a sinus augmentation procedure. J Periodontol. 2007;78:955-61.

[29] Bassil J, Senni K, Changotade S, Baroukh B, Kassis C, Naaman N, et al. Expression of MMP-2, 9 and 13 in newly formed bone after sinus augmentation using inorganic bovine bone in human. J Periodontal Res. 2011;46:756-62.

[30] Schwartz Z, Weesner T, van Dijk S, Cochran DL, Mellonig JT, Lohmann CH, et al. Ability of deproteinized cancellous bovine bone to induce new bone formation. $\mathbf{J}$ Periodontol. 2000;71:1258-69.

[31] Mordenfeld A, Hallman M, Johansson CB, Albrektsson T. Histological and histomorphometrical analyses of biopsies harvested 11 years after maxillary sinus floor augmentation with deproteinized bovine and autogenous bone. Clin Oral Implants Res. 2010;21:961-70.

[32] Yildirim M, Spiekermann H, Biesterfeld S, Edelhoff D. Maxillary sinus augmentation using xenogenic bone substitute material Bio-Oss in combination with venous blood. A histologic and histomorphometric study in humans. Clin Oral Implants Res. 2000;11:217-29. 
[33] Oliveira R, El Hage M, Carrel JP, Lombardi T, Bernard JP. Rehabilitation of the edentulous posterior maxilla after sinus floor elevation using deproteinized bovine bone: a 9-year clinical study. Implant Dent. 2012;21:422-6.

[34] Kamakura S, Sasano Y, Homma-Ohki H, Nakamura M, Suzuki O, Kagayama M, et al. Multinucleated giant cells recruited by implantation of octacalcium phosphate (OCP) in rat bone marrow share ultrastructural characteristics with osteoclasts. J Electron Microsc (Tokyo). 1997;46:397-403.

[35] Murakami Y, Honda Y, Anada T, Shimauchi H, Suzuki O. Comparative study on bone regeneration by synthetic octacalcium phosphate with various granule sizes. Acta Biomater. 2010;6:1542-8.

[36] Takami M, Mochizuki A, Yamada A, Tachi K, Zhao B, Miyamoto Y, et al. Osteoclast differentiation induced by synthetic octacalcium phosphate through receptor activator of NF-kappaB ligand expression in osteoblasts. Tissue Eng Part A. 2009; 15:3991-4000.

[37] Oreffo RO, Mundy GR, Seyedin SM, Bonewald LF. Activation of the bonederived latent TGF beta complex by isolated osteoclasts. Biochem Biophys Res Commun. 1989;158:817-23.

[38] Pfeilschifter J, Bonewald L, Mundy GR. Characterization of the latent transforming growth factor beta complex in bone. J Bone Miner Res. 1990;5:49-58.

[39] Tang Y, Wu X, Lei W, Pang L, Wan C, Shi Z, et al. TGF-beta1-induced migration of bone mesenchymal stem cells couples bone resorption with formation. Nat Med. 2009;15:757-65.

[40] Zhao C, Irie N, Takada Y, Shimoda K, Miyamoto T, Nishiwaki T, et al. Bidirectional ephrinB2-EphB4 signaling controls bone homeostasis. Cell Metab. 2006;4:111-21.

[41] Pederson L, Ruan M, Westendorf JJ, Khosla S, Oursler MJ. Regulation of bone formation by osteoclasts involves Wnt/BMP signaling and the chemokine sphingosine-1-phosphate. Proc Natl Acad Sci U S A. 2008;105:20764-9.

[42] Ryu J, Kim HJ, Chang EJ, Huang H, Banno Y, Kim HH. Sphingosine 1phosphate as a regulator of osteoclast differentiation and osteoclast-osteoblast coupling. EMBO J. 2006;25:5840-51.

[43] Takeshita S, Fumoto T, Matsuoka K, Park KA, Aburatani H, Kato S, et al. Osteoclast-secreted CTHRC1 in the coupling of bone resorption to formation. J Clin Invest. 2013;123:3914-24.

[44] de Bruijn JD, Bovell YP, Davies JE, van Blitterswijk CA. Osteoclastic resorption of calcium phosphates is potentiated in postosteogenic culture conditions. J Biomed Mater Res. 1994;28:105-12.

[45] Gilles JA, Carnes DL, Windeler AS. Development of an in vitro culture system for the study of osteoclast activity and function. J Endod. 1994;20:327-31.

[46] Gomi K, Lowenberg B, Shapiro G, Davies JE. Resorption of sintered synthetic hydroxyapatite by osteoclasts in vitro. Biomaterials. 1993;14:91-6.

[47] Yamada S, Heymann D, Bouler JM, Daculsi G. Osteoclastic resorption of biphasic calcium phosphate ceramic in vitro. J Biomed Mater Res. 1997;37:346-52.

[48] Dimitriou R, Tsiridis E, Giannoudis PV. Current concepts of molecular aspects of bone healing. Injury. 2005;36:1392-404.

[49] Jimi E, Nakamura I, Duong LT, Ikebe T, Takahashi N, Rodan GA, et al. Interleukin 1 induces multinucleation and bone-resorbing activity of osteoclasts in the absence of osteoblasts/stromal cells. Exp Cell Res. 1999;247:84-93.

[50] Katagiri T, Takahashi N. Regulatory mechanisms of osteoblast and osteoclast differentiation. Oral Dis. 2002;8:147-59. 


\section{Figure legends}

Figure 1: Scanning electron microscopy images of deproteinized bovine bone (DBB) (A, C and E) and synthetic tetrapod-shaped calcium phosphate (TetraB) (B, D and F) at increasing magnification; x100 (A and B), x1,000 (C and D) and x50,000 (E and F).

Figure 2: X-ray diffraction analysis of deproteinized bovine bone (DBB) (A) and synthetic tetrapod-shaped calcium phosphate (TetraB) (B). The XRD peaks show that the main phase of DBB is hydroxyapatite, whereas TetraB mainly consists of $\alpha$-TCP (peaks around $20-35^{\circ}$ ) and part of OCP (main peak at $4.70^{\circ}$ ) and hydroxyapatite. $(*=$ hydroxyapatite; $\#=\alpha-\mathrm{TCP} ; \mathrm{O}=\mathrm{OCP})$.

Figure 3: Survey light micrographs of undecalcified toluidine blue-stained sections of bone defects created in the trabecular region of the rat femoral epiphysis. The defects were unaugmented Sham site (A, D and G) or augmented with either deproteinized bovine bone $(\mathrm{DBB})(\mathrm{B}, \mathrm{E}$ and $\mathrm{H})$ or synthetic tetrapod-shaped calcium phosphate (TetraB) $(\mathrm{C}, \mathrm{F}$ and $\mathrm{I})$. The images show the pattern of new bone formation (NB) within the three defects at $6 \mathrm{~d}(\mathrm{~A}, \mathrm{~B}$ and C), $14 \mathrm{~d}(\mathrm{D}, \mathrm{E}$ and F) and $28 \mathrm{~d}(\mathrm{G}, \mathrm{H}$ and I). At 6d (A, D and G), there was no evident bone formation in the central region of any of the defect groups. The central regions are mainly occupied by soft tissue (ST) in the Sham sites (A), whereas those in the augmented defects appear to be mainly occupied by the DBB (B) or TetraB (C) granules. Peripherally, newly formed bone (NB) is observed in all defect types extending from the peripheral original bone (OB). The NB reveals dark staining assuming an unmineralized osteoid appearance. No major signs of inflammatory infiltrate can be detected in any of the defects. At 14d (D, E and F), a substantial amount of bone has formed, extending from the periphery of the 
defects and filling the central region in all groups. In the augmented sites (E and F), the NB in a trabecular pattern appears to fill the intergranular spaces and connect between the individual granules. In this period, the NB staining appears to be slightly lighter than at $6 \mathrm{~d}$, whereas the TetraB $(\mathrm{F})$ is even lighter, assuming a certain degree of mineralization. At $28 \mathrm{~d}$ in the Sham site $(\mathrm{G})$, the NB, observed earlier, is considerably remodeled, leaving few trabeculae of lightly stained bone bordered by bone marrow (BM) and soft tissue. The soft tissue on the top of the Sham defects reveals a downgrowth inside the defect area. In this period in the augmented sites ( $\mathrm{H}$ and $\mathrm{I})$, the NB is maintained, filling the major area of the defects, together with the granules of either substitute material. In both the DBB and TetraB, the NB extends to the top of the defects, retaining the original level of the bone at those sites. A larger amount of lightly stained mineralized bone appears to fill the intergranular spaces in the TetraB group compared with the DBB.

Figure 4: Light micrographs of undecalcified toluidine blue-stained sections of bone defects created in the trabecular region of the rat femoral epiphysis. The images show the augmented sites at $6 \mathrm{~d}$. The defects were augmented with either deproteinized bovine bone (DBB) (A, B and C) or synthetic tetrapod-shaped calcium phosphate (TetraB) (D, E and F). In this period, the newly formed bone is primarily unmineralized osteoid (Os) formed in the bone marrow (BM) in the close vicinity of granules of either type. The Os in the TetraB group reveals higher proximity and contact with the granule surfaces than that formed in the DBB group. In addition, areas of partially mineralized bone (PMB), lightly stained, are mainly detected in the TetraB-treated defects. Osteoblast seams are also observed adjacent to the Os areas in both defect groups (some are indicated by white arrowheads). 
Figure 5: Light micrographs of undecalcified toluidine blue-stained sections of bone defects created in the trabecular region of the rat femoral epiphysis. The images show the augmented sites at $14 \mathrm{~d}$. The defects were augmented with either deproteinized bovine bone (DBB) (A, B, C, D) or synthetic tetrapod-shaped calcium phosphate (TetraB) (E, F, G, H, I). In this period, the bone filling the intergranular spaces in both groups appears as a mixture of partially mineralized (PMB) and mineralized bone (MB), indicated by the lighter degree of staining and the presence of osteocytes. In the TetraB group, the MB shows a higher degree of contact with the granule surface $(\mathrm{F})$ than that in the DBB (B). Furthermore, the interface between the MB and the TetraB granules frequently reveals a blade-like appearance (indicated by white arrows in $\mathrm{H}$ ). Osteoblast seams and osteoclast-like cells are detected, in many cases in connection with the granule surfaces of either material (indicated by white and black arrowheads, respectively). Surface irregularities (resorption-like sites; indicated by black arrows), facing the osteoclast-like cells, appear in both the MB and the granule surface in the TetraB defects and only in the MB in the DBB defects.

Figure 6: Light micrographs of undecalcified toluidine blue-stained sections of bone defects created in the trabecular region of the rat femoral epiphysis. The images show the augmented sites at $28 \mathrm{~d}$. The defects were augmented with either deproteinized bovine bone (DBB) (A, B and C) or synthetic tetrapod-shaped calcium phosphate (TetraB) (D, E and F). In this period, the bone in the intergranular spaces appears to be mineralized, with numerous osteocyte lacunae indicating mature bone type (MB). The DBB and TetraB particles show a comparable degree of contact with the MB. Active remodeling sites are encountered, characterized by osteoblast seams (white arrowheads) and osteoclast-like cells (black arrowheads). The latter observation is more commonly detected in the TetraB group. 
Figure 7: Histomorphometric analysis of defects created in the trabecular region of the rat femoral epiphysis. The defects were unaugmented Sham site or augmented with either deproteinized bovine bone (DBB) or synthetic tetrapod-shaped calcium phosphate (TetraB). The analysis was performed at 6, 14 and 28d. Measurements were made of the granule area percentages, bone area percentages and the percentages of bone-granule contact. The level of statistical significance was set at $\mathrm{p}<0.05$. Statistically significant differences are indicated by symbols or small letters: $*=$ significant difference between the Sham site versus any of the augmented sites (DBB or TetraB); $\#=$ significant difference between $\mathrm{DBB}$ and TetraB; $\mathrm{a}=$ significant difference between $6 \mathrm{~d}$ and $14 \mathrm{~d}$ (for each group); $\mathrm{b}=$ significant difference between $14 \mathrm{~d}$ and $28 \mathrm{~d}$ (for each group). The results are presented as the mean percentages \pm SEM.

Figure 8: Backscatter SEM micrographs of defects created in the trabecular region of the rat femoral epiphysis. The defects were augmented with either deproteinized bovine bone (DBB) (A and $\mathrm{C}$ ) or synthetic tetrapod-shaped calcium phosphate (TetraB) (B and D). The images show selected areas of the augmented sites at $3 \mathrm{~d}$ (A and $B)$ and $6 \mathrm{~d}(\mathrm{C}$ and $\mathrm{D})$. The insert shows a higher magnification of a selected area in $(\mathrm{D}) . \mathrm{PMB}=$ partially mineralized bone; $\mathrm{OB}=$ original bone; The white arrows $(\mathrm{B}$ and insert in D) refer to direct contact between PMB and TetraB.

Figure 9: Backscatter SEM micrographs of defects created in the trabecular region of the rat femoral epiphysis. The defects were augmented with either deproteinized bovine bone (DBB) (A and $\mathrm{C}$ ) or synthetic tetrapod-shaped calcium phosphate (TetraB) (B and D). The images show selected areas of the augmented sites at $14 \mathrm{~d}$. White arrowheads refer to the interface between the particle surface and the formed bone. (OC) indicates osteocyte lacunae. 
Figure 10: Backscatter SEM (A-D) and HAADF-STEM (E and F) micrographs of defects created in the trabecular region of the rat femoral epiphysis. The defects were augmented with either deproteinized bovine bone (DBB) (A, C and E) or synthetic tetrapod-shaped calcium phosphate (TetraB) (B, D and F). The images show selected areas of the augmented sites at $28 \mathrm{~d}$. White arrowheads refer to the interface between the particle surface and the formed bone. (OC) denotes osteocyte lacuna; (C) denotes canaliculus.

Figure 11: Gene expression analysis of tissue harvested from defects created in the trabecular region of the rat femoral epiphysis. The defects were unaugmented Sham site or augmented with either deproteinized bovine bone (DBB) or synthetic tetrapodshaped calcium phosphate (TetraB). The analysis was performed at 6, 14 and $28 \mathrm{~d}$. The level of statistical significance was set at $\mathrm{p}<0.05$. Statistically significant differences are indicated by symbols or small letters: $*=$ significant difference between the Sham site versus any of the augmented sites (DBB or TetraB); \# = significant difference between DBB and TetraB; $a=$ significant difference between $3 \mathrm{~d}$ and $6 \mathrm{~d}$ (for each group); $\mathrm{b}=$ significant difference between $6 \mathrm{~d}$ and $14 \mathrm{~d}$ (for each group); $\mathrm{c}=$ significant difference between $14 \mathrm{~d}$ and $28 \mathrm{~d}$ (for each group). The results are presented as the mean \pm SEM. 


\begin{tabular}{|c|c|c|c|c|c|c|c|c|c|}
\hline & \multicolumn{3}{|c|}{ Sham } & \multicolumn{3}{|c|}{ DBB } & \multicolumn{3}{|c|}{ TetraB } \\
\hline & Genes & $(\mathbf{r})$ & (p) & Genes & (r) & (p) & Genes & (r) & (p) \\
\hline \multirow{4}{*}{$3 d$} & & & & TRAP/CatK & 0.83 & 0.04 & TRAP/CatK & 0.96 & 0.0004 \\
\hline & TNF- $\alpha / \mathrm{OC}$ & -0.94 & 0.004 & & & & & & \\
\hline & TNF- $\alpha /$ TRAP & 0.83 & 0.04 & & & & & & \\
\hline & TRAP/CatK & 0.77 & 0.04 & TRAP/CatK & 0.94 & 0.004 & TRAP/CatK & 0.89 & 0.006 \\
\hline \multirow{3}{*}{$6 \mathrm{~d}$} & & & & & & & TNF- $\alpha /$ ALP & 0.79 & 0.04 \\
\hline & & & & & & & OC/TRAP & 0.89 & 0.006 \\
\hline & & & & & & & OC/CatK & 0.79 & 0.04 \\
\hline \multirow{6}{*}{$14 d$} & TNF- $\alpha /$ IL-1 $\beta$ & 0.89 & 0.02 & TNF- $\alpha /$ IL-1 $\beta$ & 0.89 & 0.006 & TNF- $\alpha /$ IL-1 $\beta$ & 0.86 & 0.01 \\
\hline & & & & TRAP/CatK & 0.96 & 0.0004 & TRAP/CatK & 0.96 & 0.0004 \\
\hline & & & & TNF- $\alpha /$ CatK & 0.89 & 0.006 & TNF- $\alpha /$ CatK & 0.79 & 0.04 \\
\hline & & & & TNF- $\alpha /$ TRAP & 0.82 & 0.02 & TNF- $\alpha /$ TRAP & 0.86 & 0.01 \\
\hline & & & & IL-1 $\beta /$ CatK & 1 & $1 \mathrm{E}-10$ & IL-1 $\beta /$ CatK & 0.96 & 0.0004 \\
\hline & & & & IL-1 $\beta /$ TRAP & 0.96 & 0.0004 & IL-1 $\beta /$ TRAP & 0.93 & 0.003 \\
\hline \multirow{10}{*}{$28 \mathrm{~d}$} & TNF- $\alpha /$ IL-1 $\beta$ & 0.83 & 0.04 & TNF- $\alpha /$ IL- $1 \beta$ & 0.82 & 0.02 & TNF- $\alpha / \mathrm{IL}-1 \beta$ & 0.82 & 0.02 \\
\hline & TRAP/CatK & 1 & $1 \mathrm{E}-10$ & TRAP/CatK & 0.89 & 0.007 & TRAP/CatK & 0.93 & 0.002 \\
\hline & & & & ALP/OC & 0.89 & 0.007 & ALP/OC & 0.75 & 0.049 \\
\hline & TNF- $\alpha /$ ALP & 0.89 & 0.02 & & & & TNF- $\alpha /$ ALP & 0.93 & 0.002 \\
\hline & & & & & & & TNF- $\alpha /$ CatK & 0.79 & 0.04 \\
\hline & & & & & & & IL-1 $\beta /$ CatK & 0.79 & 0.04 \\
\hline & & & & & & & IL-1 $\beta /$ TRAP & 0.82 & 0.02 \\
\hline & & & & ALP/CatK & 0.89 & 0.007 & ALP/CatK & 0.79 & 0.03 \\
\hline & OC/TRAP & 0.89 & 0.02 & & & & & & \\
\hline & OC/CatK & 0.89 & 0.02 & & & & & & \\
\hline
\end{tabular}

Table I: Correlation analysis of genes expressed in samples retrieved from unaugmented Sham defects or from defects augmented with either deproteinized bovine bone

(DBB) or synthetic tetrapod-shaped calcium phosphate (TetraB). The analysis was performed at 3, 6, 14 and $28 \mathrm{~d}$ after augmentation. Spearman correlation coefficients (r) and level of significance (p-values) are presented. 


\section{Figure 1}
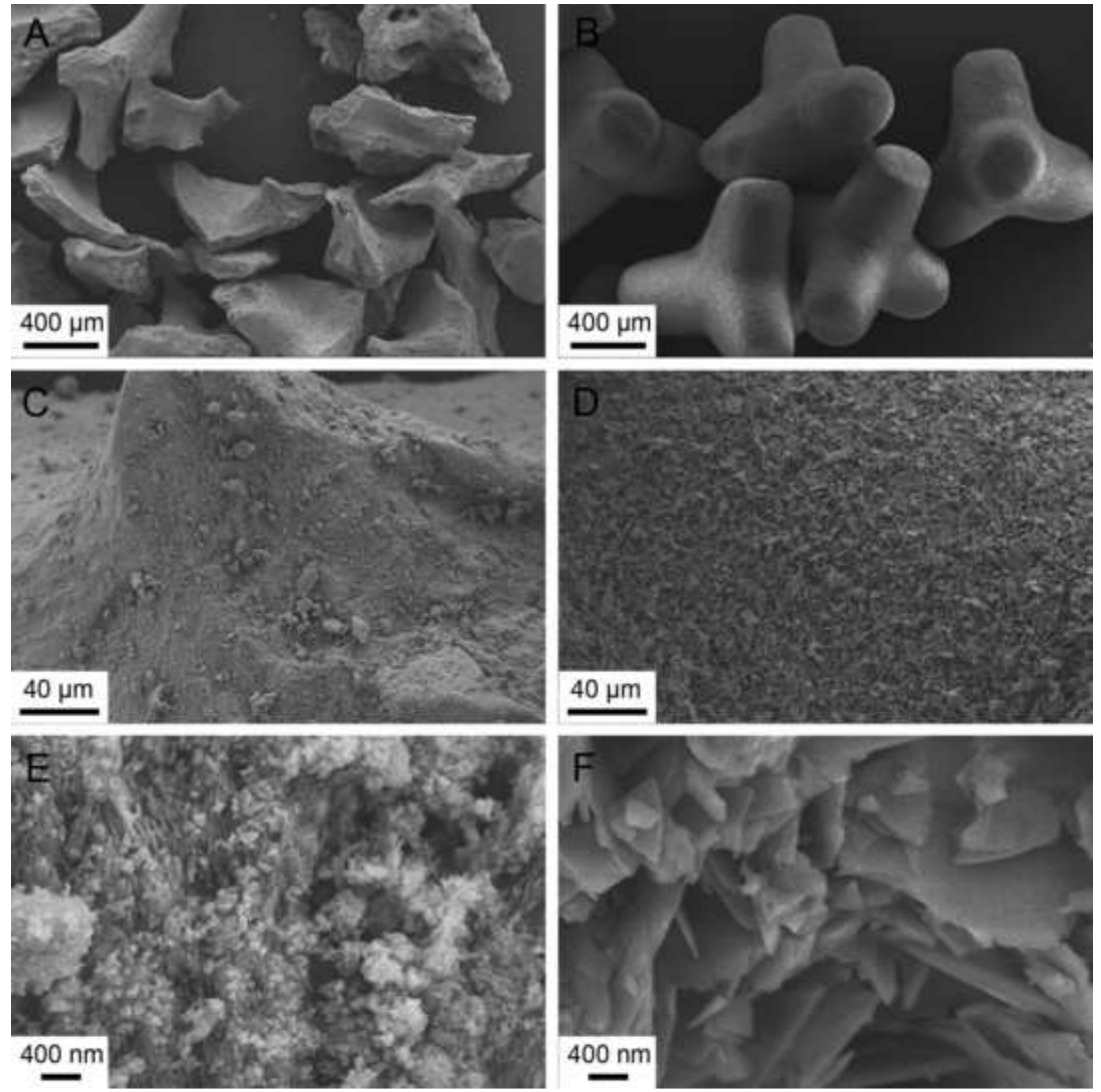

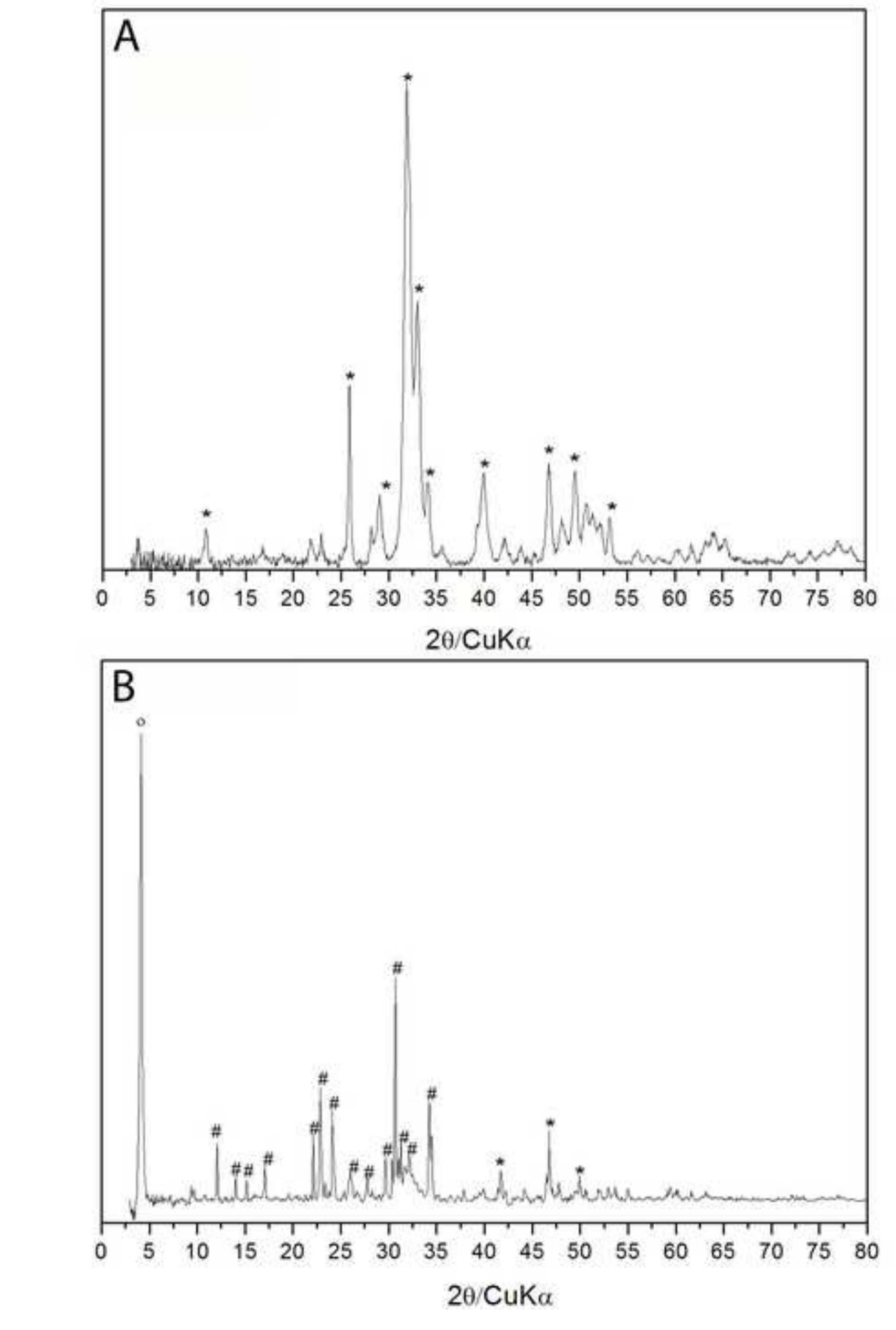

Figure 2

.




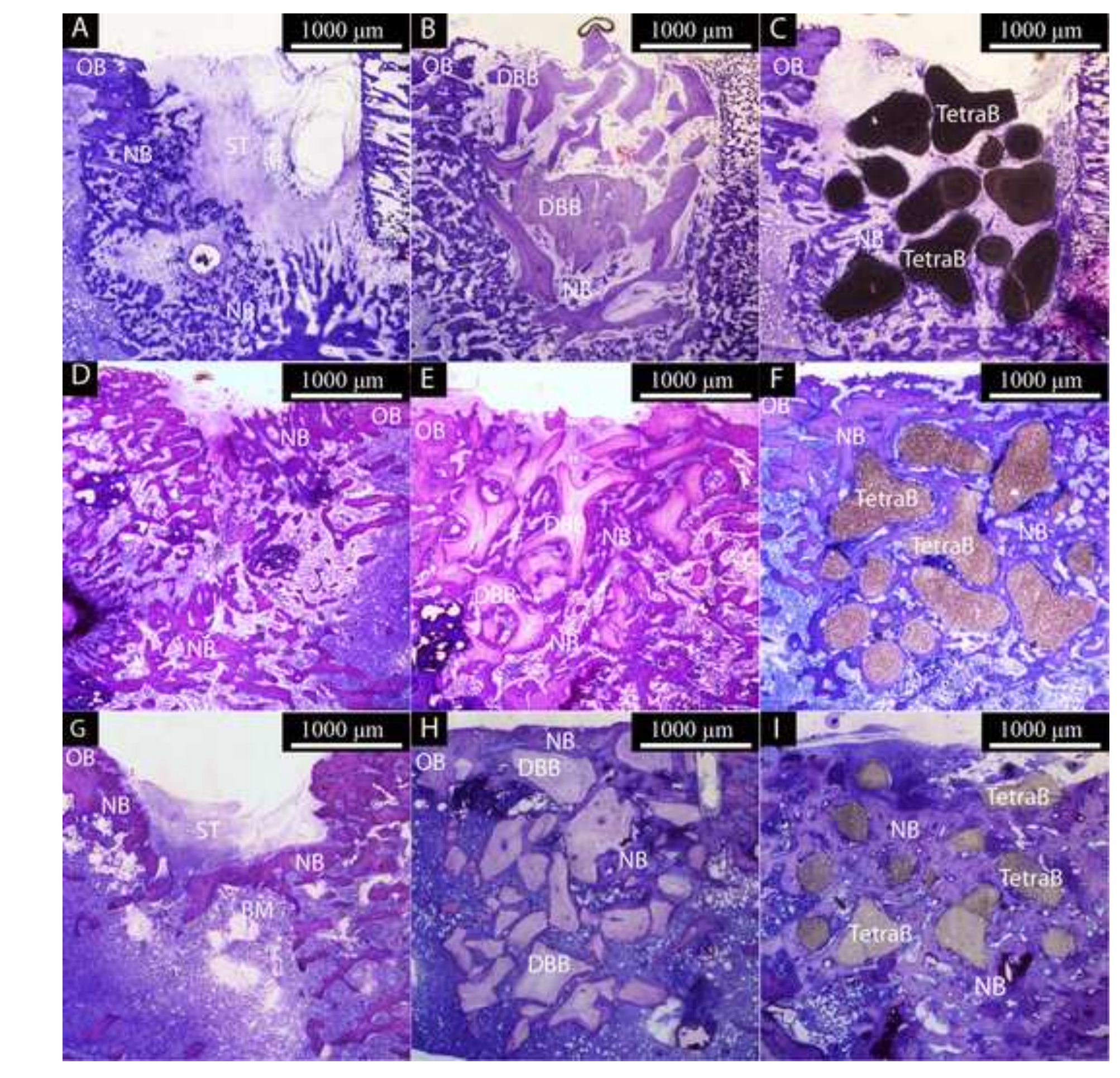

$G$
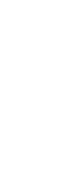

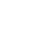

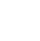

\section{Figure 3}




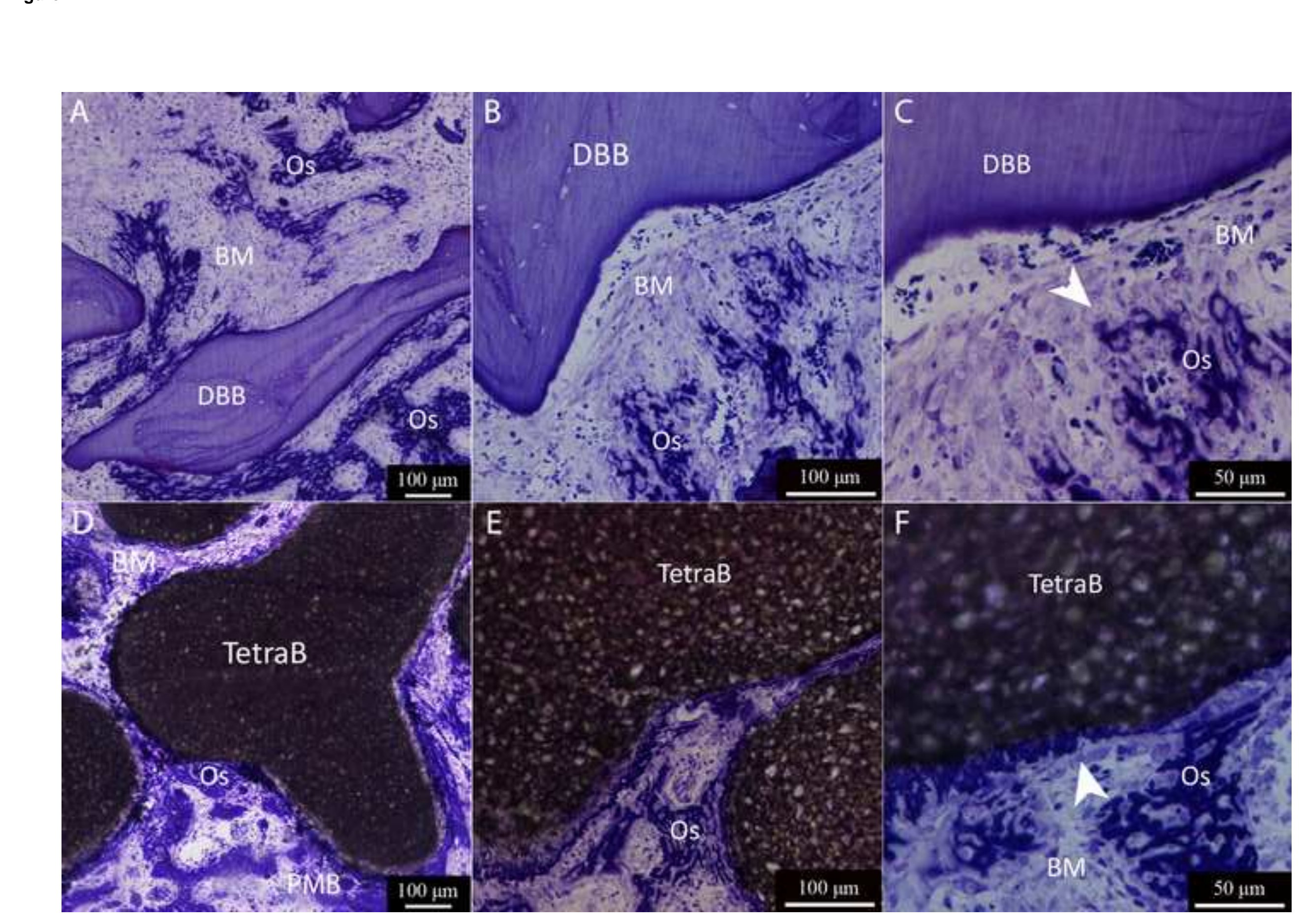




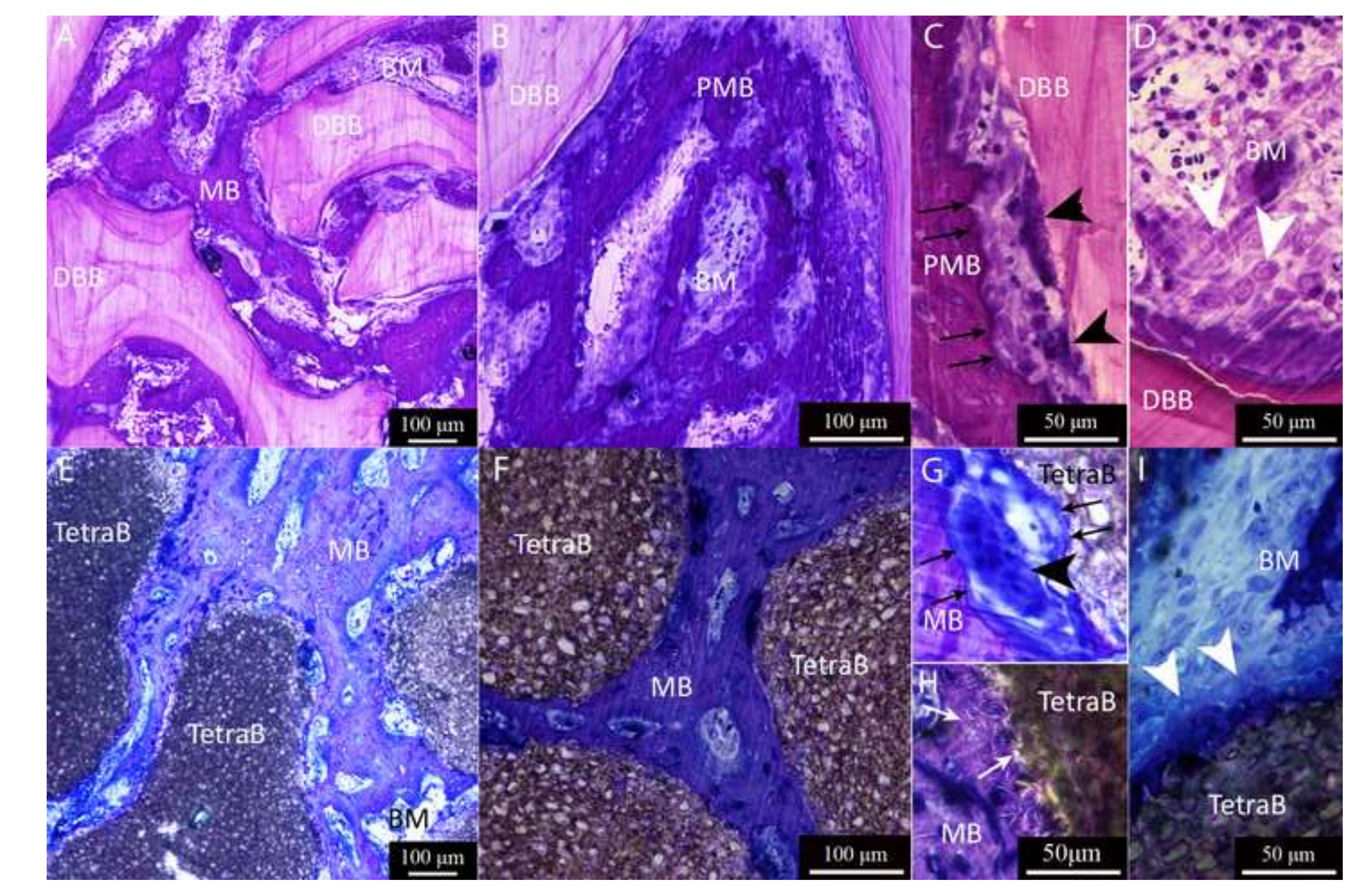

\author{
-
}




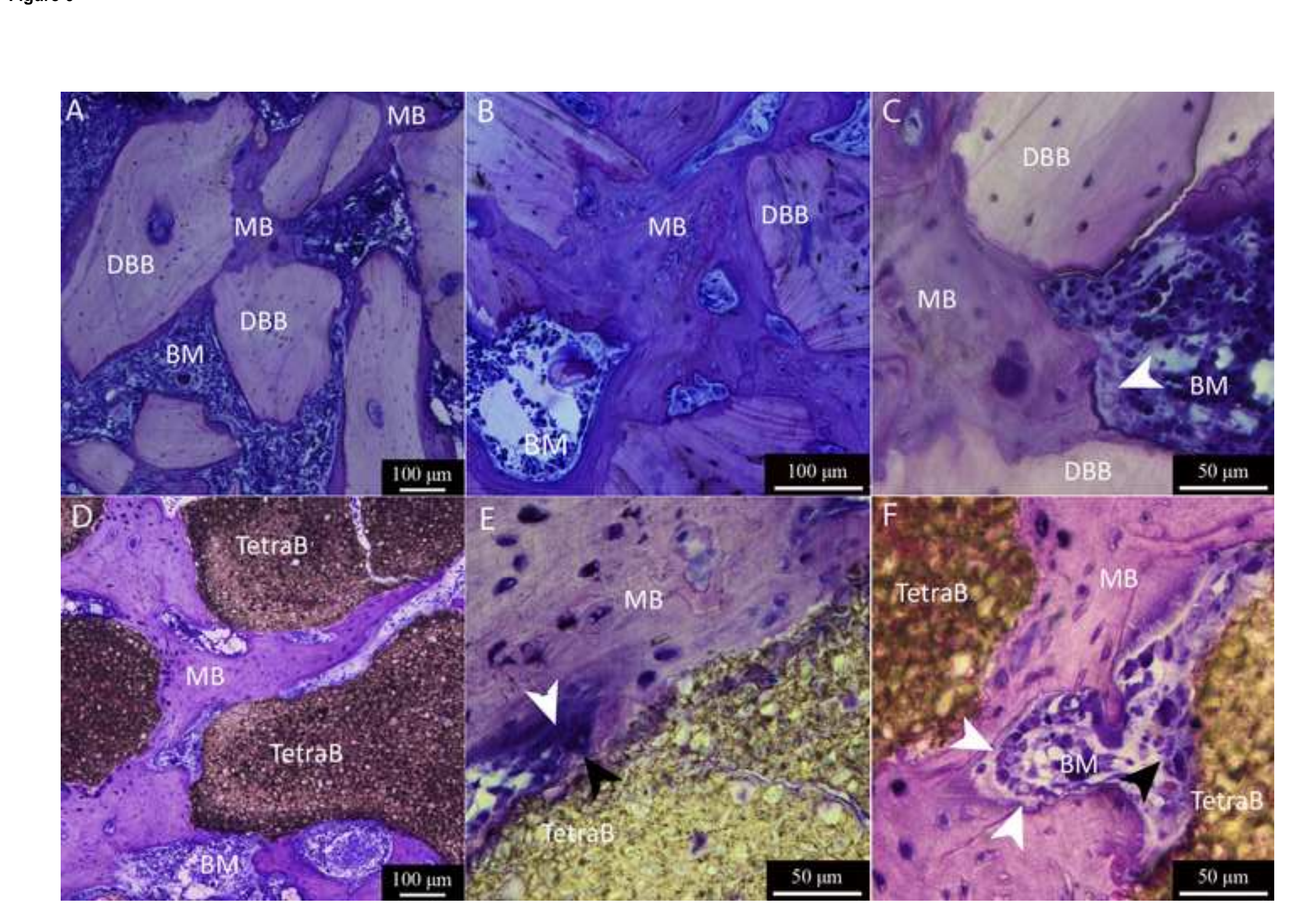

. 

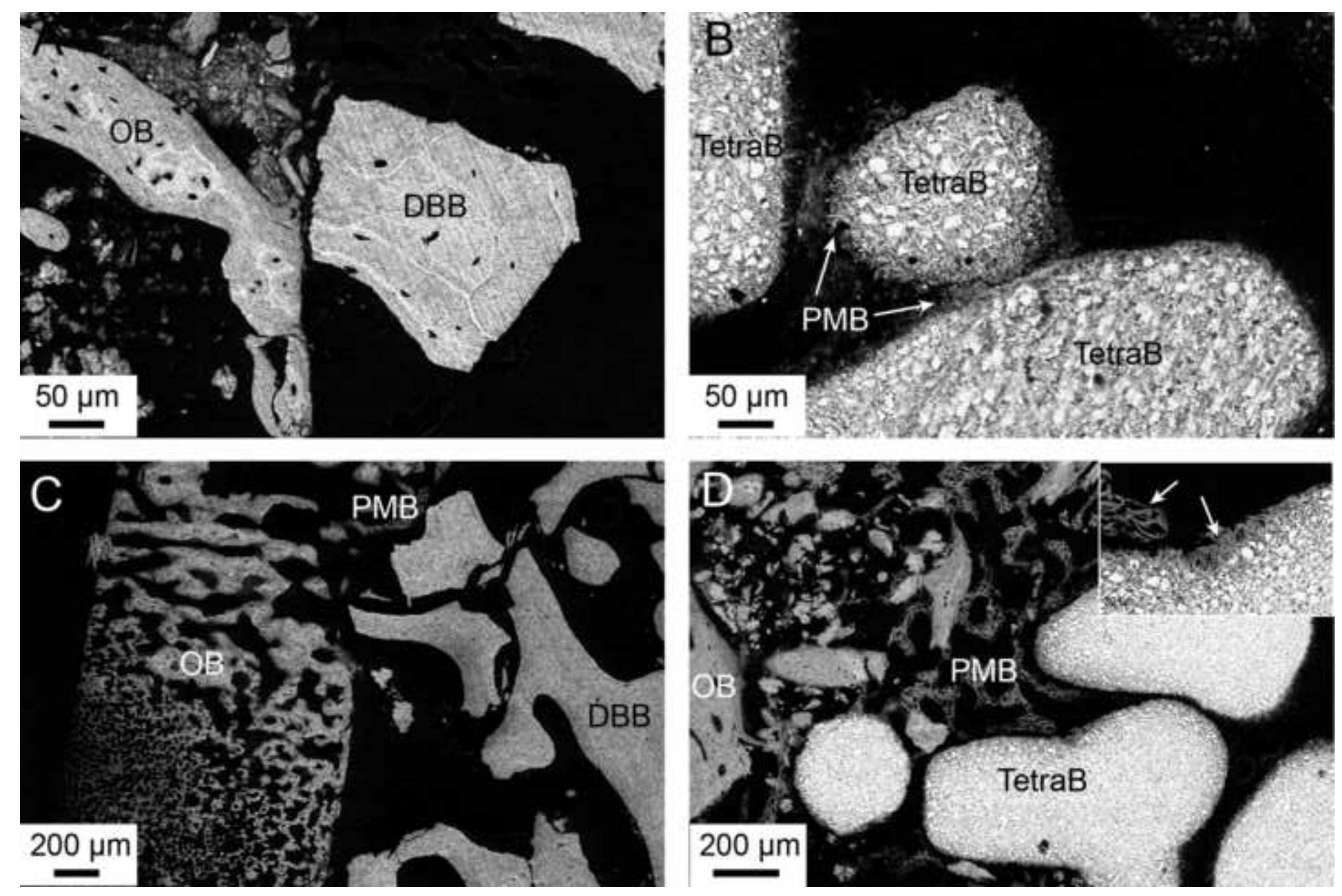

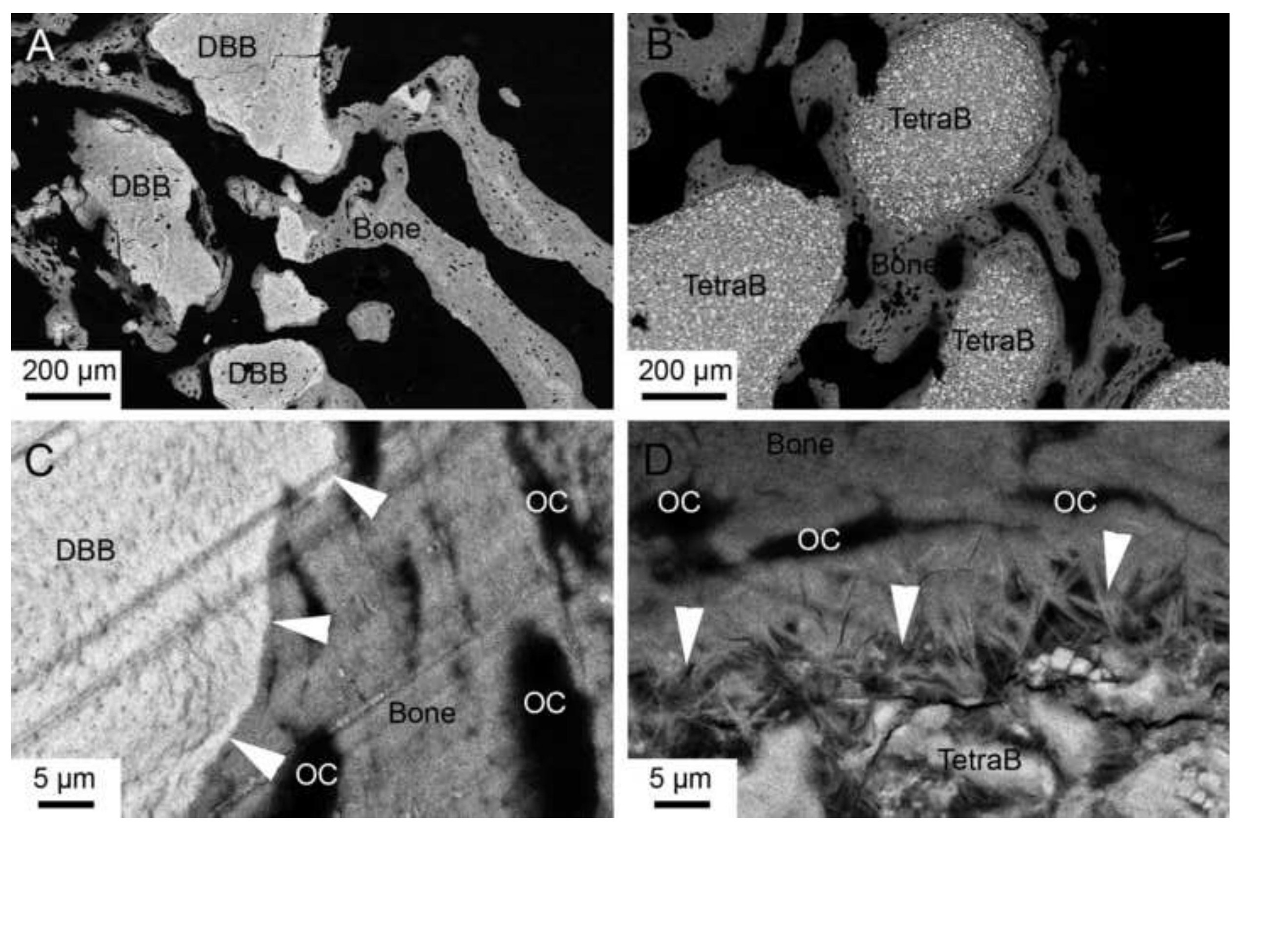

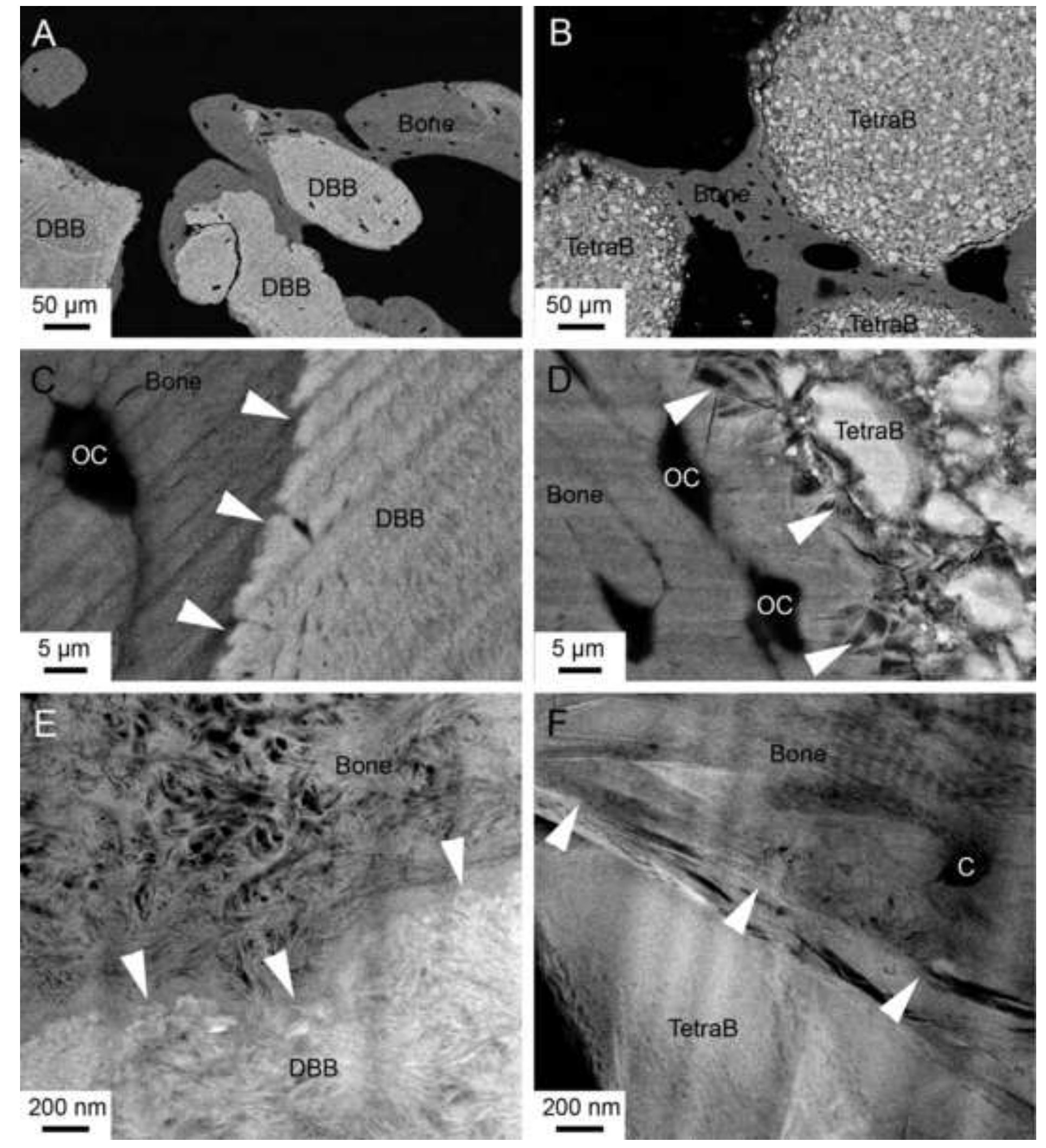

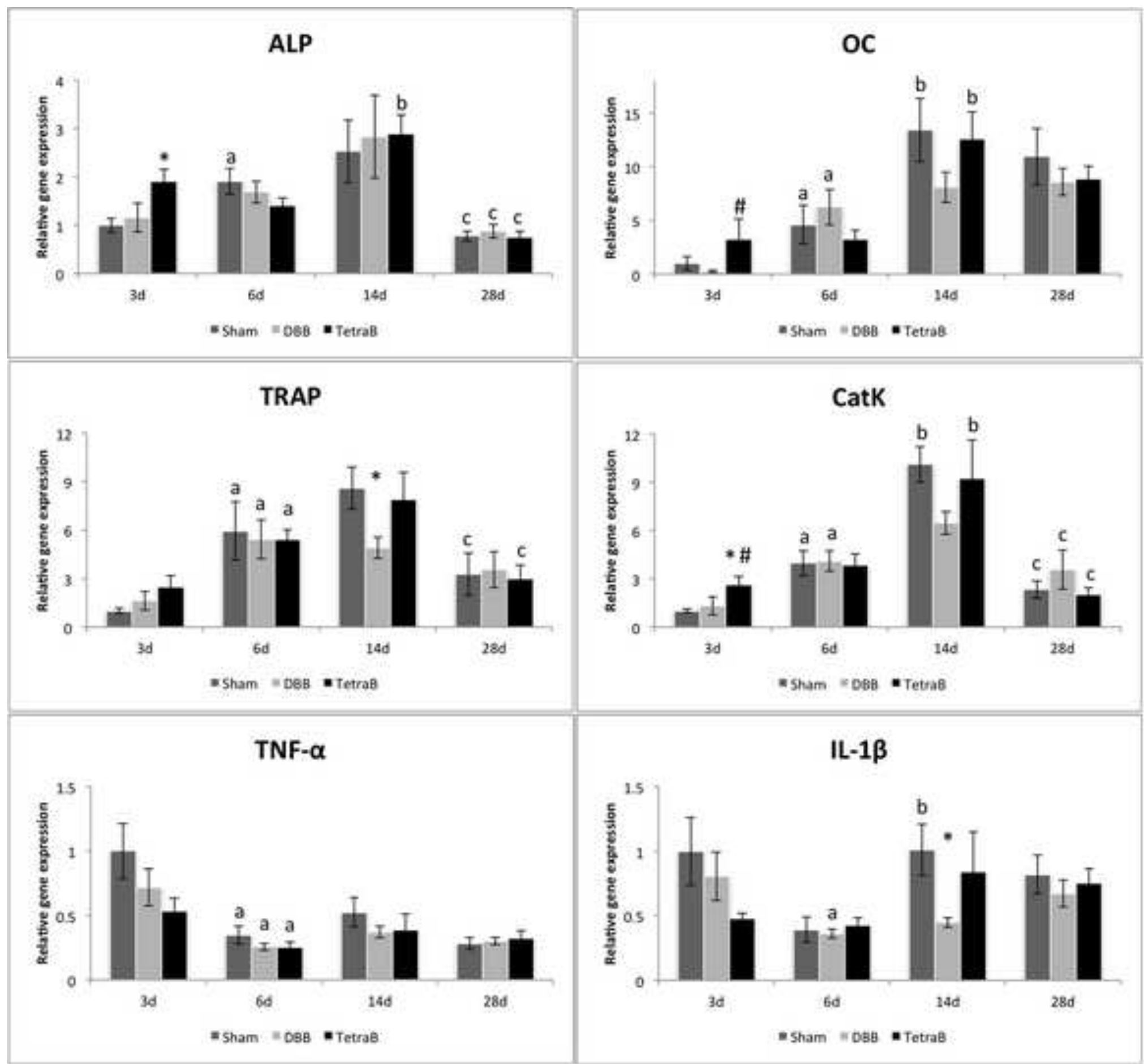